\title{
CONFLICT MANAGEMENT STYLES IN RELATION TO DEMOGRAPHICS
}

\author{
GÜLER İSLAMOĞLU \\ Marmara University
}

\author{
DENIZ BÖRய̈ \\ Marmara University
}

\author{
MELEK BİRSEL \\ Marmara University
}

\begin{abstract}
Research carried out has implied that conflict management style varies according to gender (Baxter and Shephard, 1978), and position in the hierarchy (Fagenson, 1990; Kanter, 1977), education, professional experience, and tenure (Hendelet al., 2005). In this study, data were collected using the Conflict Management Style inventory of Rahim (1983) and Dreu et al. (2001). The sample consisted of 984 employees working in different organizations and in different sectors in Istanbul. In order to test the variance between different groups, a t-test and ANOVA test were conducted. The results of the analysis revealed that conflict management style differs in relation to tenure and position.
\end{abstract}

Key words: conflict, conflict management style, competiting, collaborating, accomodating, avoiding, compromise, demographics.

\section{DEMOGRAFIK ÖZELLIKLERE GÖRE ÇATIŞMA ÇÖZME YÖNTEMLERI}

ÖZET

Yapılan araştırmalar çatışmayı ele alış tarzlarının cinsiyete (Baxter ve Shephard, 1978), hiyeraşik kademedeki pozisyona (Fagenson, 1990; Kanter, 1977), eğitim, iş hayatındaki tecrübe ve kıdeme

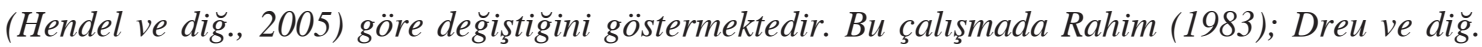
nin (2001) Çatışmayı Ele Alış Tarzı envanteri kullanılarak veri toplanmıştır. Örneklemi İstanbul'da farklı sektör ve kurumlarda çalışan 984 kişi oluşturmaktadır. Gruplar arasındaki farklılı̆̆ı ortaya koymak için t-test ve ANOVA uygulanmıştır. Analiz sonuçları çatışmayı ele alış tarzlarının hiyeraşik kademedeki pozisyona ve kıdeme göre farklılaştığını ortaya koymuştur.

Anahtar kelimeler: çatışma, çatışmayı ele alış tarzı, rekabet, işbirliği, uyma, kaçınma, uzlaşma, demografik değişkenler.

* Güler İslamoğlu is an Associate Professor in the Department of Business Administration at Marmara University, Göztepe Campus, 34722, Ziverbey, Istanbul, Turkey. E-mail: gislamoglu@ marmara.edu.tr

** Deniz Börü is an Associate Professor in the Department of Business Administration at Marmara University, Anadoluhisarı Campus, 34815, Beykoz, Istanbul, Turkey. E-mail: denizboru@ marmara.edu.tr

*** Melek Birsel is an Assistant Professor in the Department of Business Administration at Marmara University, Göztepe Campus, 34722, Ziverbey, Istanbul, Turkey. E-mail: mbirsel@ marmara.edu.tr 
Conflict is a common issue in our everyday lives. Caused by disagreement in goals, motivations, or actions between two parties that can be real or only perceived to exist, conflict is seen as a perceived incongruity of interests (Taylor and Moghaddam, 1994). Whether it results due to a difference of opinions, harsh words or direct action to solve competing goals, conflict has the potential to occur in many domains of our lives. Conflict is considered to be the normal and inevitable consequence of social and organizational life (Coser, 1956). Dahrendorf (1959) and Mosca (1939) claim further that no human society or social group exists that does not possess some conflict between the wishes or intent of individuals, while Kahn et al. (1964) state that "one might well make a case for interpreting some conflict as essential for the continued development of mature and competent human beings." Conflict can occur in any aspect of human interaction.

Conflict typically has been viewed as an indication of organizational and interpersonal dysfunction. Mayo (1945) contends that organizations should attempt to avoid conflict wherever possible. The human relations movement, which emphasizes the potential personal and organizational costs of conflict, also believes conflict must be purged from the organizational setting (Kelly, 1970; Litterer, 1966). There are conflict theorists, however, who believe that some degree of interpersonal and organizational conflict can serve useful functions (Lewis, 1976; O’Connor, 1978; Schmidt and Tannenbaum, 1960). Deutsch (1971) states that "conflict is often part of the process of testing and assessing one's self and as such, may be highly enjoyable as one experiences the pleasure of full and active use of one's capacities." This interest shows that conflict can be seen in all kinds of situations in organizations and in social life (Cosier and Ruble, 1981; Miles, 1980), and some studies show that managers spend a serious amount of their time dealing with conflict (Pondy, 1992; Pullhamus, 1991; Thomas and Schmidt, 1976).

Many factors such as high diversity, uncertain work environments and organizational interdependencies cause conflict in organizations (Amason, 1996; Amason et al., 1995; Jameson, 1999; Pondy, 1992; Wall and Callister, 1995). Since conflict is inevitable and accepted as a major facet of organizational life, conflict resolution within organizations is a topic that receives significant attention in the academic literature and in the professional environment (De Dru and Weingart, 2003; Jameson, 1999; Jehn, 1997; Pearson et al., 2002; Rahim, 2000, 2001, 2002; Wall and Callister, 1995).

As a result, conflict management has recently become a major subfield of organizational behavior. This might be due to the trend that stresses the greater acceptance of conflict as an organizational phenomena, and as a result, concern over its management. Since there is a great need for workplace harmony and productivity (Chen and Tjosvold, 2002; Tjosvold and Sun, 2002), managing conflict is becoming vital. Although conflict traditionally has been considered to be destructive, De Dreu and Van de Vliert (1997) suggest that constructive conflicts may provide some positive outcomes, such as improving group creativity and effectiveness. A credible reason for the continuing interest in conflict management is that the ability to resolve conflicts is considered an important skill for managers (McKenna and Richardson, 1995). Some scholars (e.g., Cosier and Schwenk, 1990; Jehn, 1994; Priem et al., 1995) even believe that conflicts could be functional while the quality of decisions would improve as a result of intense debate. Rahim (1992) indicates that organizational conflict is considered as a legitimate, inevitable, and possibly a positive indicator of effective organization management.

In spite of increasing interest in conflict management, the influence of demographic variables on conflict management has been overlooked. The studies conducted analyzing the relationship between conflict management styles and demographic variables have involved mostly gender and have yielded 
inconsistent results (McKenna and Richardson, 1995; Çetin and Hacıfazlıŏ̆lu, 2004; Rahim, 1983, Bedell and Sistrunk,1973). Therefore, this study includes other demographic variables such as education, marital status, having children, position, tenure in the current organization and the total tenure. The relationship between these demographic varibles and conflict management styles has been tested while these relationships are thought to be important for improving workplace relations.

After reviewing the theoretical grounding and developing the research question related with demographic variables and conflict management styles, the research method utilized in this study will be explained detailing the instrument, sampling and the procedure. Then, the empirical results with a discussion of the findings will be elaborated. Finally, the managerial implications of the research will be presented.

\section{LITERATURE}

Conflict is defined as "tension between two or more social entities (individuals, groups, or larger organizations) which arises from incompatibility of actual or desired responses" (Raven and Kruglanski, 1970). Another definition for conflict is "a process in which one party perceives that its interests are being opposed or negatively affected by another party" (Wall and Callister, 1995).

According to Roloff (1987: 496), "organizational conflict occurs when members engage in activities that are incompatible with those of colleagues within their network, members of other collectivities or unaffiliated individuals who utilize the services or products of the organization." Conflict can be conceptualized as an interactive process manifested in incompatibility, disagreement, or dissonance within or between social entities (i.e., individual, group, organization, etc.). Conflict may occur when (Rahim, 2002):

1. A party is required to carry on an activity that is not congruent with his or her needs or interests.

2. A party prefers certain behavior, the satisfaction of which is incompatible with another person's implementation of his or her preferences.

3. A party wants some mutually desirable resource that is in short supply; therefore the needs of everyone may not be fully met.

4. A party holds attitudes, values, skills, and goals that are prominent in directing his or her behavior, but are perceived to be restricted by the attitudes, values, skills, and goals of the other(s).

5. Two parties have partially exclusive behavioral preferences in relation to their joint actions.

6. Two parties are interdependent in the performance of functions or activities.

Different approaches might be utilized for dealing with conflict. This difference in approach might be due to a variety of factors such as culture, personality, type of conflict and the demographics of the parties involved in conflict (Kozan, 1997). Differences in cultural artifacts, such as language, religion, geographical location as well as in cultural beliefs and values, are also identified as some of the key causes of the differences in conflict management styles (Kozan, 1997).

There are different styles of behavior to handle conflict (Drory and Ritu, 1997). Blake and Mouton (1964) identified a two-dimensional model based on "concern for people" and "concern for 
production" for classifying styles of conflict management. Thomas (1976) made an adaptation of the two-dimensional model and suggested the dimensions of "assertiveness" and "cooperativeness" in classifying the styles. Rahim (1983) refers to the dimensions as "concern for self" versus "concern for others" in classifying the styles of managing conflicts into five types: Integrating, Dominating, Obliging, Avoiding, and Compromising.

The model of conflict management styles initially presented by Blake and Mouton (1964) and further developed by Kilmann and Thomas (1975) and Rahim (1983) identifies the following five conflicthandling styles (Rahim, 1992; Rahim and Magner, 1995). The styles for handling conflict vary according to two basic dimensions: "concern for self" and "concern for others." The integrating/ collaborating style refers to high concern for self as well as the other party involved in the conflict. It involves collaboration between parties. The obliging/accomodating style refers to low concern for self and high concern for others. It is concerned with smoothing over differences and focusing on areas of agreement. The dominating/competing style involves a high concern for self and a low concern for the other party involved in the conflict. It has been described as forcing one's viewpoint at the expense of others. The avoiding style involves low concern for self as well as the other party. It is concerned with withdrawing from the conflict situation. The compromising style refers to moderate concern for self as well as the party involved in the conflict. It is concerned with give-and-take or sharing the search for a middle-ground solution.

Conflict management skills are very significant for being able to function effectively at any level within the organization. With the increase in the number of females moving into positions requiring decision-making, the possible effect of gender differences in the ability to manage conflict comes to mind (Powell, 1988). Some are skeptical about women's ability to adjust to managerial roles and responsibilities while the managerial role is associated with masculine rather than feminine characteristics (Brenner et al., 1989; Powell and Butterfield, 1979).

However, the literature on the conflict handling styles of males and females is inconsistent. For example, in a study of Singaporean managers (McKenna and Richardson, 1995), men were found to use the compromising style more than women, while women were more inclined to use the avoiding style. While Rahim (1983) found that women were more likely to use cooperative styles (such as obliging and integrating), Bedell and Sistrunk (1973) suggested that women were more competitive.

On the other hand, some research suggests that males and females occupying similar positions behave in much the same way in managing conflict (Eagly and Johnson, 1990; Korabik, Baril and Watson, 1993; Powell, 1988). Some research, on the other hand, suggests that women have a more cooperative approach to conflict than men (Rahim, 1983; Rubin and Brown, 1975). In a study conducted by Green et al. (2002), it was found that conflict management styles differed according to biological sex and gender role. The feminine group used more avoiding styles compared to male and androgynous groups, whereas male groups tend to be more dominating. Similarly, Portello and Long (1994) have proved in their studies that gender roles play an important part in the choice of conflict resolution styles. For example, individuals with a masculine gender role are found to be more aggressive and, hence, would utilize a dominating or competing style. On the other hand, feminine individuals are found to be more cooperative and thus, favor the avoiding style (Baxter and Shepherd, 1978). The integrating (or collaborating), obliging (or accommodating) and compromising styles appear to be applicable to androgynous individuals (Bern and Lenney, 1976; Portello and Long, 1994). Cook 
(1985). Furthermore, Brewer et al. (2002) argue that although biological sex may be associated with conflict management styles, gender role is the determinant of individuals' reaction to conflicts. This line of reasoning is supported by the belief that masculine and feminine characteristics are learned, especially when individuals progress in an organization.

In a study conducted by Çetin and Hacıfazlioğlu (2004), among university academics and high school teachers, gender was also found to be important in determining conflict management style although different findings were obtained among the academics and teachers included in the study. Male academics were found to be more tolerant and flexible whereas female teachers were found to be less offensive toward their colleagues.

In the study by Çetin and Hacifazlioglu (2004), it was also found out that besides gender, age, experience and faculty, and subject variables played vital roles in teachers' and academics' choice of appropriate conflict management styles. Age was observed to be one of the main important variables that affect conflict management styles. As teachers and academics get older, they become more flexible and constructive in their dialogues with their peers. This was proved by the more frequent use of a collaboration style by the older subjects.

The experience variable was found not to create a difference among teachers' conflict management styles in this study. Academics within the range of 11-20 years of experience were found to choose a compromising approach more, in contrast to academics within the range of one to five years and 21 and above years of experience. This could be related to the career path they are on. It could be thought that reaching a stable point in their careers might be a factor leading them to behave in a more compromising way. Results also revealed that academics' faculties affected their conflict management styles. Academics in the faculty of education were found to use accommodation and collaboration more than academics in the faculty of business administration. This finding is indirectly supported by another study conducted by Cornille et al. (1999). The study conducted to compare the conflict management styles of teachers and business managers with those of their peers. It found that there are significant differences in the conflict management styles used by teachers when compared with those of business managers.

In some of the previous studies conducted, conflict management styles were found to differ according to position. Fagenson (1990) and Kanter (1977) have suggested that the individual's approach to conflict management may differ according to the position held in the organizational hierarchy. Results of a three-factor MANOVA conducted by Temkin and Cummings (1986), on the other hand, showed no significant differences in conflict management style related with position, gender and tenure.

A study carried out among Israeli nurse managers in general hospitals has found that most of the demographic characteristics were not correlated with conflict management styles. However, tenure in position was found to have influence on the choice of collaborating in conflict management. The more tenure a head nurse had in position, the more frequent she used the collaborating style for conflict management (Hendel et al., 2005).

Based on different research findings related with conflict management styles and demographical variables, this study aims to investigate whether the conflict management styles of individuals differ according to gender, marital status, having children, tenure in the current organization, total tenure in 
work life, educational background, and position of the individual. Therefore, the research question of the study is "Does the conflict management style of an individual differ according to demographic variables?"

\section{METHOD}

The research aims to find out whether certain demographic variables affect conflict management styles. The independent variables taken for the study are:

Gender

Marital Status

Having Children

Education

Total Tenure in Worklife

Tenure in the Current Organization

Position of the Individual

The dependent variable is the conflict management styles, namely:

Accommodation

Competition

Collaboration

Avoiding

Compromise

\section{Instruments}

The instrument consists of two parts. The first part asks the individuals questions related to their demographics (gender, marital status, having children, education, total tenure in worklife, tenure in the current organization and position of the individual). The professions and the departments of the employees were not taken as demographic variables because the organizations that were accessed did not have similar departmentalization and the professions ranged on a large scale such that it might not be possible to make classification. Similarly, age, place of birth, sector of the organization, number of children, variables related to education such as faculty, high school graduated from, public or private university studied, city of residence, number of income earners, and income level have not been included as demographic variables because the participants were selected on the basis of convenience of access. With this type of sampling, it would be hard to make classifications with these variables in order to put them into analysis since it would be difficult to have the same number of participants in each categorization.

The second part of the instrument includes items related to conflict management style. The inventory utilized for the study is the DUTCH test developed by Rahim (1983), and Dreu et al. (2001) and used by Kuşçuluoğlu (2004) in her study.

The instrument was originally designed to measure five orthogonal dimensions of conflict management patterns: collaboration (five items), such as "I try to integrate my ideas with the other party" and "I collaborate with the other party to come up with decisions acceptable to us;" competition (five items), for example, "I am generally firm in pursuing my side of the issue" and "I argue my case with the 
other party to show the merits of my position;" compromising (five items), such as "I usually propose a middle ground for breaking deadlocks" and "I use 'give and take' so that a compromise can be made;" accommodation (five items), for example, "I often go along with the suggestions of the other party" and "I try to satisfy the expectations of the other party;" and avoiding (five items), such as "I try to stay away from disagreements with the other part" and "I usually avoid open discussion of my differences with the other party." The respondents rated each statement on a six-point scale $(1=$ never, $6=$ always).

\section{Sampling}

The participants of the study were selected on the basis of convenience of access. The questionnaires were distributed in the organizations that had agreed to be included in the study, therefore the participation was voluntary. Since the participation in the study was voluntary and the questionnaires were distributed to the organizations that had convenience of access, the sampling method is convenience sampling. The participants of the study were $(\mathrm{N}=984)$ working in different organizations in different sectors in Istanbul. As only a limited number of organizations agreed to be included in the study, the sample number was low and the results could not be generalized to the whole population. According to self-reported demographics, the distribution of the sample is given in Table 1.

Table 1

Frequency Distribution of Demographic Variables

\begin{tabular}{|c|c|c|c|c|c|c|c|}
\hline & & $\mathbf{N}$ & $\%$ & & & $\mathbf{N}$ & $\%$ \\
\hline \multirow{2}{*}{ Gender } & Female & 440 & 44.7 & \multirow{3}{*}{ Position } & Upper level & 228 & 23.2 \\
\hline & Male & 544 & 55.3 & & Middle Level & 623 & 63.3 \\
\hline \multirow{2}{*}{$\begin{array}{l}\text { Marital } \\
\text { Status }\end{array}$} & Married & 499 & 50.7 & & First Line & 133 & 13.5 \\
\hline & Single & 485 & 49.3 & \multirow{3}{*}{$\begin{array}{l}\text { Total Work } \\
\text { Tenure }\end{array}$} & 1-5 Years & 388 & 39.4 \\
\hline \multirow{2}{*}{$\begin{array}{l}\text { Having } \\
\text { Children }\end{array}$} & $\begin{array}{l}\text { Have } \\
\text { Children }\end{array}$ & 420 & 42.7 & & 6-15 Years & 340 & 34.6 \\
\hline & $\begin{array}{l}\text { Have No } \\
\text { Children }\end{array}$ & 564 & 57.3 & & 16-+ Years & 256 & 26.0 \\
\hline \multirow{3}{*}{ Education } & $\begin{array}{l}\text { High } \\
\text { School }\end{array}$ & 248 & 25.2 & \multirow{3}{*}{$\begin{array}{l}\text { Tenure in } \\
\text { Current } \\
\text { Organizations }\end{array}$} & 1-5 Years & 616 & 62.6 \\
\hline & University & 591 & 60.1 & & 6-15 Years & 275 & 27.9 \\
\hline & Master & 145 & 14.7 & & 16-+ Years & 93 & 9.5 \\
\hline
\end{tabular}

In this study, the tenure in work life and the tenure in the current organization were asked as openended questions while the rest of the demographics were multiple choice questions. The results obtained showed that both tenures varied across a wide range:

The tenure in work life varies between 1 and 43 years (mean 10.55 years)

The tenure in the current organization is between 1 and 40 years (mean 6.26 years) 
The researchers categorized the tenures as given in Table 1 in order to be able to make comparisons in the analyses.

Questionnaires were distributed to the employees in different organizations. A total of 1023 subjects participated in the study by answering the complete questionnaire. However, due to invalid and incomplete answers, the total number of the participants was 984 .

\section{Procedure}

Participation in the study was voluntary. Questionnaires were distributed to participants by visiting them in their offices. The completed surveys were recollected during a two week period. The questionnaire included a cover letter in which the researchers asked the participants not to write their names since participation was kept confidential. 984 questionnaires were properly answered. The response rate was $96 \%$.

\section{RESULTS}

\section{Reliability and Factor Analyses}

The SPSS program was used to make analyses at the 0.05 significance level. A reliability analysis was conducted for the conflict management style inventory utilized in this study. The instrument was found to be satisfactorily reliable since the cronbach alfa value was equal to 0.7982 .

A factor analysis was conducted to find out the subcomponents of the conflict management style inventory by using the method of principle component. The results of the factor analysis yielded four factors as opposed to the original five factors. The five items measuring the "compromise" factor were not included in the study due to low factor loadings and low reliability. One item of the "competition" factor and one item of the "avoidance" factor were deleted because they lowered the reliability of the total factor.

For the instrument identifying the conflict management styles, the KMO Measure of Sampling Adequacy was found to be .806 . This value indicates that the items of instrument were homogenous and that estimating the variance of each variable in the correlation matrix by all of the other variables in the matrix is significantly high, so these items were appropriate for factor analysis. The value of Bartlett's test of sphericity (Chi- Square:5126,447; df: 66; p:0.000) indicates that the computed factor analysis was significant. Twenty-five items were collected under four factors which have an explaining power of $56.890 \%$ of the total variation. The factors with their loadings and reliabilities are given in Table 2.

\section{Statistical Analyses}

In this study, t-tests and the ANOVA test were utilized to test the differences between groups with different demographics in relation to conflict management style.

The results of the analyses revealed that except for position there is no relationship between demographic variables and conflict management style. The results for t-test analyses are given in Table 3 and the results for the ANOVA analyses are given in Table 4. 
Table 2

Factors of Conflict Management Style

\begin{tabular}{lc}
\hline FACTOR $1:$ COLLABORATION & Mean: \\
\hline $\begin{array}{l}\mathbf{2 . 6 1 1 4} \\
\text { Q: .7609 }\end{array}$ & Factor contr. \\
$\begin{array}{l}\text { 9. Trying to find the best solution for all parties by letting everybody } \\
\text { express his/her opinion and discussing different points of view. }\end{array}$ & .747 \\
$\begin{array}{l}\text { 14. Trying to find a solution that meets the needs and interests of both } \\
\text { parties. }\end{array}$ & .704 \\
$\begin{array}{l}\text { 19. Trying to find the best solution for all the parties involved. } \\
\text { 24. Cooperating with the other parties to find the solution that meets each } \\
\text { party's expectations. }\end{array}$ & .693 \\
4. Confronting conflict openly and trying to find a solution that meets \\
each party's needs.
\end{tabular}

FACTOR 2 : ACCOMMODATION

$\begin{array}{lrrr}\alpha: .7623 & \text { Mean.: } & \% \text { var : } 14.617 & \text { Factor contr. }\end{array}$

$\begin{array}{ll}\text { 8. Letting the other party realize his/her own objectives and interests. } & .769\end{array}$

3. Conforming to the solution brought by the other party. $\quad .730$

23. Trying to meet the expectations of the other party. $\quad .710$

13. Trying to realize the other party's desires. $\quad .694$

18. Giving up one's own wants to meet the other party's desires. .598

\begin{tabular}{cccc}
\hline \multicolumn{1}{l}{ FACTOR 3 : COMPETITION } & & \\
\hline$\alpha: .7910$ & Mean.: & \%var : 14.469 & Factor contr.
\end{tabular}

11. I do whatever is needed to get the best result for myself. $\quad .829$

21. Insisting that one's point of view is right $\quad .785$

6. Trying to have the other party accept one's solution to the problem. $\quad 740$

16. Trying to get one's own needs at any cost. $\quad .699$

FACTOR 4 : AVOIDANCE

$\begin{array}{lrrr}\alpha: .7304 & \text { Mean.: } & \text { \%var :12 .846 } & \text { Factor contr. }\end{array}$

25. I keep the disagreements to myself in order to prevent negative $\quad .744$ feelings.

$\begin{array}{ll}\text { 15. I try not to confront with the other party in relation to conflict. } & .741 \\ \text { 5. I try not to interfere into conflict. } & .694\end{array}$

20. I avoid discussing the conflict issue openly with the other party. 684

Kaiser-Meyer-Olkin Measure of Sampling Adequacy: .806

Approx. Chi-Square: 5126.447 df: 153 Sig. : .000

Total Variation : $56.890 \%$ 
Table 3

Results of t-Test Analyses

\begin{tabular}{|c|c|c|c|c|c|c|}
\hline $\begin{array}{c}\text { CONFLICT } \\
\text { MANAGEMENT STYLES }\end{array}$ & Variables & Mean & $\begin{array}{c}\text { Stand. } \\
\text { Dev. }\end{array}$ & $\mathrm{t}$ & df & Sig. \\
\hline \multicolumn{7}{|c|}{ GENDER } \\
\hline \multirow{2}{*}{ COLLABORATION } & FEMALE & 2.6345 & .88587 & \multirow{2}{*}{.742} & \multirow{2}{*}{933.811} & \multirow{2}{*}{.458} \\
\hline & MALE & 2.5926 & .87314 & & & \\
\hline \multirow{2}{*}{ ACCOMMODATION } & FEMALE & 3.6800 & .98793 & \multirow{2}{*}{.770} & \multirow{2}{*}{955.673} & \multirow{2}{*}{.441} \\
\hline & MALE & 3.6301 & 1.03535 & & & \\
\hline \multirow{2}{*}{ COMPETITION } & FEMALE & 3.0903 & 1.11546 & \multirow{2}{*}{1.469} & \multirow{2}{*}{946.575} & \multirow{2}{*}{.142} \\
\hline & MALE & 2.9844 & 1.13732 & & & \\
\hline \multirow{2}{*}{ AVOIDANCE } & FEMALE & 3.7097 & 1.03109 & \multirow{2}{*}{1.000} & \multirow{2}{*}{952.230} & \multirow{2}{*}{.318} \\
\hline & MALE & 3.6425 & 1.06895 & & & \\
\hline \multicolumn{7}{|c|}{ MARITAL STATUS } \\
\hline \multirow{2}{*}{ COLLABORATION } & MARRIED & 2.6385 & .88755 & \multirow{2}{*}{.981} & \multirow{2}{*}{981.939} & \multirow{2}{*}{.327} \\
\hline & SINGLE & 2.5835 & .86944 & & & \\
\hline \multirow{2}{*}{ ACCOMMODATION } & MARRIED & 3.6794 & 1.02159 & \multirow{2}{*}{.845} & \multirow{2}{*}{981.809} & \multirow{2}{*}{.399} \\
\hline & SINGLE & 3.6247 & 1.00686 & & & \\
\hline \multirow{2}{*}{ COMPETITION } & MARRIED & 3.0556 & 1.12544 & \multirow{2}{*}{672} & \multirow{2}{*}{980.859} & \multirow{2}{*}{.501} \\
\hline & SINGLE & 3.0072 & 1.13179 & & & \\
\hline \multirow{2}{*}{ AVOIDANCE } & MARRIED & 3.6779 & 1.05903 & \multirow{2}{*}{.162} & 001740 & $072+2$ \\
\hline & SINGLE & 3.6670 & 1.04617 & & 901.140 & .012 \\
\hline & HA & IG CHII & REN & & & \\
\hline COLU AOP ATIOU & $\begin{array}{c}\text { HAVE } \\
\text { CHILDREN }\end{array}$ & 2.6581 & .90380 & 1400 & 076725 & $152+2>$ \\
\hline CULLADURATION & $\begin{array}{l}\text { HAVE NO } \\
\text { CHILDREN }\end{array}$ & 2.5766 & .85861 & 1.429 & $8 / 0.155$ & .155 \\
\hline & $\begin{array}{l}\text { HAVE } \\
\text { CHILDREN }\end{array}$ & 3.6843 & 1.05525 & & & \\
\hline 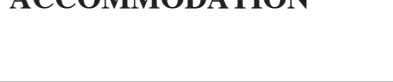 & $\begin{array}{l}\text { HAVE NO } \\
\text { CHILDREN }\end{array}$ & 3.6287 & .98281 & .041 & 000.100 & . 401 \\
\hline 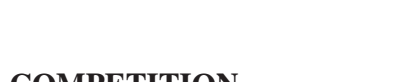 & $\begin{array}{c}\text { HAVE } \\
\text { CHILDREN }\end{array}$ & 3.0244 & 1.13689 & 176 & 006650 & $060 \rightarrow+2 \rightarrow$ \\
\hline COAITE TIITUN & $\begin{array}{l}\text { HAVE NO } \\
\text { CHILDREN }\end{array}$ & 3.0372 & 1.12276 & $-.1 / 0$ & 090.050 & .000 \\
\hline 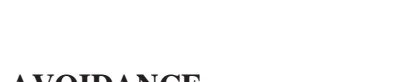 & $\begin{array}{l}\text { HAVE } \\
\text { CHILDREN }\end{array}$ & 3.6732 & 1.07209 & 010 & $006(6)$ & 006 \\
\hline AVUIDAIVCE & $\begin{array}{l}\text { HAVE NO } \\
\text { CHILDREN }\end{array}$ & 3.6720 & 1.03808 & . 010 & 000.000 & .900 \\
\hline
\end{tabular}


Table 4

Results of ANOVA Analyses

\begin{tabular}{|c|c|c|c|c|c|c|c|}
\hline \multirow{2}{*}{$\begin{array}{c}\text { CONFLICT } \\
\text { MANAGEMENT } \\
\text { STYLES } \\
\end{array}$} & \multirow{2}{*}{\multicolumn{2}{|c|}{ VARIABLES }} & \multicolumn{2}{|c|}{ ANOVA } & \multicolumn{3}{|c|}{ MULTIPLE COMPARISONS (Scheffe) } \\
\hline & & & $\mathrm{F}$ & Sig. & Mean Diff. & $\begin{array}{l}\text { Stand. } \\
\text { Dev. }\end{array}$ & Sig. \\
\hline \multicolumn{8}{|c|}{ EDUCATION } \\
\hline \multirow{6}{*}{ COLLABORATION } & \multirow{2}{*}{ High School } & University & \multirow{6}{*}{.991} & \multirow{6}{*}{.372} & -.01613 & .06648 & .971 \\
\hline & & Master & & & -.12096 & .09186 & .421 \\
\hline & \multirow{2}{*}{ University } & High School & & & .01613 & .06648 & .971 \\
\hline & & Master & & & -.10483 & .08143 & .437 \\
\hline & \multirow{2}{*}{ Master } & High School & & & .12096 & .09186 & .421 \\
\hline & & University & & & .10483 & .08143 & .437 \\
\hline \multirow{6}{*}{ ACCOMMODATION } & \multirow{2}{*}{ High School } & University & \multirow{6}{*}{2.550} & \multirow{6}{*}{.079} & -.12484 & .07661 & .266 \\
\hline & & Master & & & .05445 & .10586 & .876 \\
\hline & \multirow{2}{*}{ University } & High School & & & .12484 & .07661 & .266 \\
\hline & & Master & & & .17929 & .09384 & .162 \\
\hline & \multirow{2}{*}{ Master } & High School & & & -.05445 & .10586 & .876 \\
\hline & & University & & & -.17929 & .09384 & .162 \\
\hline \multirow{6}{*}{ COMPETITION } & \multirow{2}{*}{ High School } & University & & & -.08032 & .08540 & .643 \\
\hline & & Master & & & -.10022 & .11800 & .697 \\
\hline & Univercity & High School & & & .08032 & .08540 & .643 \\
\hline & University & Master & .535 & .586 & -.01990 & .10461 & .982 \\
\hline & & High School & & & .10022 & .11800 & .697 \\
\hline & Master & University & & & .01990 & .10461 & .982 \\
\hline & Hirh Sohol & University & & & -.06187 & .07966 & .740 \\
\hline & High School & Master & & & -.08391 & .11006 & .748 \\
\hline & & High School & & & .06187 & .07966 & .740 \\
\hline AVOIDANCE & University & Master & .392 & .676 & -.02204 & .09757 & .975 \\
\hline & & High School & & & .08391 & .11006 & .748 \\
\hline & Master & University & & & .02204 & .09757 & .975 \\
\hline & & TAL TENL & E IN W & ORKI & IFE & & \\
\hline & $=\mathbf{Y}$ & 6-15 Years & & & -.01113 & .06528 & .986 \\
\hline & 1-5 Years & 16-+ Years & & & .08199 & .07075 & .511 \\
\hline COU I APOD ATION & $6-15$ Years & 1-5 Years & & & .01113 & .06528 & .986 \\
\hline COLLABORATION & 6-15 Years & 16-+ Years & .947 & .388 & .09313 & .07271 & .441 \\
\hline & $16+$ Years & 1-5 Years & & & -.08199 & .07075 & .511 \\
\hline & 16-+ Years & 6-15 Years & & & -.09313 & .07271 & .441 \\
\hline & 1-5 Years & 6-15 Years & & & -.05098 & .07521 & .795 \\
\hline & 1-5 Years & 16-+ Years & & & -.18736 & .08152 & .072 \\
\hline ACCOMМODUTIOU & & 1-5 Years & 2701 & & .05098 & .07521 & .795 \\
\hline ACCOMMODATION & 6-15 Years & 16-+ Years & 2.701 & .068 & -.13638 & .08378 & .266 \\
\hline & & 1-5 Years & & & .18736 & .08152 & .072 \\
\hline & 16-+ Years & 6-15 Years & & & .13638 & .08378 & .266 \\
\hline & & 6-15 Years & & & -.07560 & .08379 & .666 \\
\hline & 1-5 Years & 16-+ Years & & & -.14550 & .09082 & .278 \\
\hline COMPFTITION & $6-15$ Years & 1-5 Years & 1311 & 270 & .07560 & .08379 & .666 \\
\hline COMPETTION & 6-15 Years & 16-+ Years & 1.311 & .210 & -.06990 & .09333 & .756 \\
\hline & & 1-5 Years & & & .14550 & .09082 & .278 \\
\hline & 16-+ Years & 6-15 Years & & & .06990 & .09333 & .756 \\
\hline & 15 Voom & 6-15 Years & & & -.07959 & .07819 & .596 \\
\hline & 1-5 Years & 16-+ Years & & & -.07195 & .08476 & .698 \\
\hline AYOID ANCF & & 1-5 Years & 621 & & .07959 & .07819 & .596 \\
\hline AVOIDANCE & 6-15 Years & 16-+ Years & .621 & .537 & .00764 & .08710 & .996 \\
\hline & $16+$ Years & 1-5 Years & & & .07195 & .08476 & .698 \\
\hline & $16-+$ Years & 6-15 Years & & & -.00764 & .08710 & .996 \\
\hline
\end{tabular}


Table 4 (continued)

TOTAL TENURE IN CURRENT ORGANIZATION

\begin{tabular}{|c|c|c|c|c|c|c|c|}
\hline \multirow{6}{*}{ COLLABORATION } & \multirow{2}{*}{ 1-5 Years } & 6-15 Years & \multirow{6}{*}{.398} & \multirow{6}{*}{.672} & .05686 & .06376 & .672 \\
\hline & & 16-+ Years & & & .02062 & .09781 & .978 \\
\hline & \multirow{2}{*}{ 6-15 Years } & 1-5 Years & & & -.05686 & .06376 & .672 \\
\hline & & 16-+ Years & & & -.03624 & .10546 & .943 \\
\hline & \multirow{2}{*}{ 16-+ Years } & 1-5 Years & & & -.02062 & .09781 & .978 \\
\hline & & 6-15 Years & & & .03624 & .10546 & .943 \\
\hline \multirow{6}{*}{ ACCOMMODATION } & \multirow{2}{*}{ 1-5 Years } & 6-15 Years & \multirow{6}{*}{.839} & \multirow{6}{*}{.433} & .03675 & .07357 & .883 \\
\hline & & 16-+ Years & & & -.12074 & .11285 & .564 \\
\hline & \multirow{2}{*}{ 6-15 Years } & 1-5 Years & & & -.03675 & .07357 & .883 \\
\hline & & 16-+ Years & & & -.15750 & .12168 & .433 \\
\hline & \multirow[b]{2}{*}{ 16-+ Years } & 1-5 Years & & & .12074 & .11285 & .564 \\
\hline & & 6-15 Years & & & .15750 & .12168 & .433 \\
\hline \multirow{6}{*}{ COMPETITION } & \multirow{2}{*}{ 1-5 Years } & 6-15 Years & \multirow{6}{*}{2.820} & \multirow{6}{*}{.060} & -.16550 & .08167 & .129 \\
\hline & & 16-+ Years & & & -.20304 & .12528 & .269 \\
\hline & \multirow{2}{*}{ 6-15 Years } & 1-5 Years & & & .16550 & .08167 & .129 \\
\hline & & 16-+ Years & & & -.03754 & .13509 & .962 \\
\hline & \multirow{2}{*}{ 16-+ Years } & 1-5 Years & & & .20304 & .12528 & .269 \\
\hline & & 6-15 Years & & & .03754 & .13509 & .962 \\
\hline \multirow{6}{*}{ AVOIDANCE } & \multirow{2}{*}{ 1-5 Years } & 6-15 Years & \multirow{6}{*}{.158} & & -.02015 & .07637 & .966 \\
\hline & & 16-+ Years & & & .05070 & .11715 & .911 \\
\hline & $6-15$ Years & 1-5 Years & & 854 & .02015 & .07637 & .966 \\
\hline & 6-15 Years & 16-+ Years & & .854 & .07085 & .12632 & .854 \\
\hline & & 1-5 Years & & & -.05070 & .11715 & .911 \\
\hline & 16-+ Years & 6-15 Years & & & -.07085 & .12632 & .854 \\
\hline & & PO & TION & & & & \\
\hline & & Middle Level & & & .03511 & .06797 & .875 \\
\hline & Upper L. & First Line & & & -.11241 & .09582 & .503 \\
\hline COU ADOD & Gidu $\mathbf{I}$ & Upper Level & 1556 & 211 & -.03511 & .06797 & .875 \\
\hline COLLABORATION & Middle L. & First Line & 1.556 & .211 & -.14752 & .08388 & .214 \\
\hline & & Upper Level & & & .11241 & .09582 & .503 \\
\hline & First L. & Middle Level & & & .14752 & .08388 & .214 \\
\hline & & Middle Level & & & .00104 & .07829 & 1.000 \\
\hline & Upper L. & First Line & & & .25614 & .11036 & .068 \\
\hline АССОММОDАTIOУ & Middle I & Upper Level & & & -.00104 & .07829 & 1.000 \\
\hline ACCOMMODATION & Middle L. & First Line & 3.666 & .026 & $.25510(*)$ & .09661 & .031 \\
\hline & & Upper Level & & & -.25614 & .11036 & .068 \\
\hline & First L. & Middle Level & & & $-.25510(*)$ & .09661 & .031 \\
\hline & & Middle Level & & & .08485 & .08612 & .616 \\
\hline & Upper L. & First Line & & & $-.49452(*)$ & .12140 & .000 \\
\hline COMPFTUTION & & Upper Level & & & -.08485 & .08612 & .616 \\
\hline COMPETITION & Middle L. & First Line & 14.879 & .000 & $-.57937(*)$ & .10628 & .000 \\
\hline & Firct I & Upper Level & & & $.49452(*)$ & .12140 & .000 \\
\hline & First L. & Middle Level & & & $.57937(*)$ & .10628 & .000 \\
\hline & & Middle Level & & & $-.19964(*)$ & .08117 & .049 \\
\hline & Upper L. & First Line & & & .00768 & .11442 & .998 \\
\hline & & Upper Level & & & $.19964(*)$ & .08117 & .049 \\
\hline AVOIDANCE & Middle L. & First Line & 4.262 & .014 & .20732 & .10017 & .118 \\
\hline & & Upper Level & & & -.00768 & .11442 & .998 \\
\hline & First L. & Middle Level & & & -.20732 & .10017 & .118 \\
\hline
\end{tabular}

* The mean difference is significant at the .05 level. 
The results reveal that:

There is a statistically significant difference between middle level managers and first line managers in relation to accommodation style. The means illustrate that middle level managers (mean: 3.6867) utilize accommodation style more than first line managers (mean: 3.4316).

There is a statistically significant difference between first line managers, upper level and middle level managers in relation to competition style. The means reveal that first line managers (mean: 3.5132) utilize this style more than upper level managers (mean: 3.0186 ) and middle level managers (mean: 2.9338).

There is a statistically significant difference between middle level managers and upper level managers in relation to avoidance style. The means show that middle level managers (mean: 3.7468 ) use avoidance style more than upper line managers (mean: 3.5471).

For the collaboration style, no difference was found between the positions.

Since differences were found between different positions in relation to conflict management styles, the analyses were conducted to test if conflict management style differs according to demographics (gender, education, marital status of employees, tenure) with different positions.

Table 5

Comparison of Different Genders with Different Positions in Relation to Conflict Management Styles

\begin{tabular}{|c|c|c|c|c|c|c|c|}
\hline \multirow{2}{*}{$\begin{array}{c}\text { Conflict } \\
\text { Management Styles }\end{array}$} & \multirow{2}{*}{\multicolumn{2}{|c|}{ Position }} & \multicolumn{2}{|c|}{ ANOVA } & \multicolumn{3}{|c|}{ Multiple Comparisons (Scheffe) } \\
\hline & & & $\mathbf{F}$ & Sig. & Mean Diff. & $\begin{array}{c}\text { Stand. } \\
\text { Dev. }\end{array}$ & Sig. \\
\hline \multicolumn{8}{|c|}{ FEMALE } \\
\hline \multirow{2}{*}{ Accomodation } & \multirow{2}{*}{3} & 1 & \multirow{2}{*}{8.831} & \multirow{2}{*}{0.00} & $-.5173 *$ & .1562 & .004 \\
\hline & & 2 & & & $-.5519 *$ & .1328 & .000 \\
\hline Competition & 3 & 2 & 4.296 & 0.14 & $.4370 *$ & .1514 & .016 \\
\hline Avoidance & 3 & 2 & 5.504 & 0.04 & $-.4593 *$ & .1396 & .005 \\
\hline \multicolumn{8}{|c|}{ MALE } \\
\hline \multirow{2}{*}{ Competition } & \multirow{2}{*}{3} & 1 & \multirow{2}{*}{11.211} & \multirow{2}{*}{0.00} & $.5616^{*}$ & .1669 & .004 \\
\hline & & 2 & & & $.7053^{*}$ & .1492 & .000 \\
\hline
\end{tabular}

* The mean difference is significant at the .05 level.

1: Upper level, 2: Middle level, 3: First line 


\section{The Analyses Conducted for Different Demographics with Different Positions}

When the analyses are examined in terms of demographics (gender, education, marital status of employees and tenure) with different position, the findings revealed that there is no significant differences between different positions with different education, marital status and tenure except between positions with gender (Table 5).

Females

There is a statistically significant difference between first line, upper level, and middle level female managers in relation to accommodation style. The means reveal that middle level female managers (mean: 3.7701) utilize this style more than upper level female managers (mean: 3.7355) and first line female managers (mean: 3.2182 ).

There is a statistically significant difference between middle level and first line female managers in relation to competition style. The means show that first line female managers (mean: 3.4583) use competition style more than middle level female managers (mean: 3.0214).

There is a statistically significant difference between middle level and first line female managers in relation to avoidance style. The means show that middle level female managers (mean: 3.8078) use avoidance style more than first line female managers (mean: 3.3485 ).

Males

There is a statistically significant difference between upper level, middle level, and first line male managers in relation to competition style. The means show that first line male managers (mean: 3.5672) use competition style more than middle level male managers (mean: 2.8618) and upper level male managers (mean: 3.0056).

\section{Comparison of Different Positions with Demographics}

Also, the analyses were conducted to test whether the conflict management style differs according to positions with different demographics (gender, education, marital status of employees, tenure ).

There is a statistically significant difference between female and male employees working at the upper level in relation to collaboration style. Female employees working at the upper level use accommodation style (mean: 2.7742) more than male employees (mean 2.5111).

There is also a statistically significant difference between female and male employees working on the first line in relation to accommodation and avoidance styles. Male employees working on the first line (mean: 3.6418) use accommodation style more than first line female managers (mean: 3.2182) and male employees working on the first line (mean: 3.7276) use avoidance style more than first line female managers (mean: 3.3485) (Table 6). 
Table 6

Comparison of Different Positions with Gender in Relation to Conflict Management Styles

\begin{tabular}{|c|c|c|c|c|c|c|}
\hline $\begin{array}{c}\text { CONFLICT } \\
\text { MANAGEMENT } \\
\text { STYLES }\end{array}$ & GENDER & Mean & $\begin{array}{c}\text { Stand. } \\
\text { Dev. }\end{array}$ & $\mathbf{t}$ & df & Sig. \\
\hline \multicolumn{7}{|c|}{ UPPER LEVEL } \\
\hline \multirow{2}{*}{ COLLABORATION } & FEMALE & 2.7742 & .81038 & \multirow{2}{*}{2.333} & \multirow{2}{*}{207.142} & \multirow{2}{*}{.02} \\
\hline & MALE & 2.5111 & .87372 & & & \\
\hline \multirow{2}{*}{ ACCOMMODATION } & FEMALE & 3.7355 & .92179 & \multirow{2}{*}{.614} & \multirow{2}{*}{212.722} & \multirow{2}{*}{.540} \\
\hline & MALE & 3.6548 & 1.04622 & & & \\
\hline \multirow{2}{*}{ COMPETITION } & FEMALE & 3.0376 & 1.01947 & \multirow{2}{*}{.225} & \multirow{2}{*}{208.880} & \multirow{2}{*}{.823} \\
\hline & MALE & 3.0056 & 1.11614 & & & \\
\hline \multirow{2}{*}{ AVOIDANCE } & FEMALE & 3.6694 & .98574 & \multirow{2}{*}{1.498} & \multirow{2}{*}{208.246} & \multirow{2}{*}{.136} \\
\hline & MALE & 3.4630 & 1.07313 & & & \\
\hline \multicolumn{7}{|c|}{ MIDDLE LEVEL } \\
\hline \multirow{2}{*}{ COLLABORATION } & FEMALE & 2.5537 & .88398 & \multirow{2}{*}{-.759} & \multirow{2}{*}{595.989} & \multirow{2}{*}{.448} \\
\hline & MALE & 2.6076 & .87711 & & & \\
\hline \multirow{2}{*}{ ACCOMMODATION } & FEMALE & 3.7701 & 1.00738 & \multirow{2}{*}{1.838} & \multirow{2}{*}{606.352} & \multirow{2}{*}{.067} \\
\hline & MALE & 3.6181 & 1.05030 & & & \\
\hline \multirow{2}{*}{ COMPETITION } & FEMALE & 3.0214 & 1.14486 & \multirow{2}{*}{1.734} & \multirow{2}{*}{596.744} & \multirow{2}{*}{.083} \\
\hline & MALE & 2.8618 & 1.13967 & & & \\
\hline \multirow{2}{*}{ AVOIDANCE } & FEMALE & 3.8078 & 1.01901 & \multirow{2}{*}{1.323} & 608067 & 186 \\
\hline & MALE & 3.6966 & 1.07264 & & 000.001 & .100 \\
\hline & & RST LII & & & & \\
\hline COU U ROP ATION & FEMALE & 2.7818 & .96251 & 642 & 128581 & 520 \\
\hline COLLADUNA TION & MALE & 2.6806 & .85142 & .042 & 120.004 & .522 \\
\hline АCCOMMODATION & FEMALE & 3.2182 & .87265 & 2685 & 130430 & 009 \\
\hline 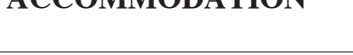 & MALE & 3.6418 & .94615 & $-2.00 J$ & 150.459 & .000 \\
\hline СОМРЕТІTIOY & FEMALE & 3.4583 & 1.06028 & -612 & 130070 & 542 \\
\hline & MALE & 3.5672 & .98913 & -.012 & 100.012 & בד \\
\hline AVOIDANCF & FEMALE & 3.3485 & 1.07496 & 2001 & 130312 & 038 \\
\hline AVOADAIVCE & MALE & 3.7276 & 1.01479 & -2.091 & 150.012 & .0J0 \\
\hline
\end{tabular}

* The mean difference is significant at the .05 level.

There is no statistically significant difference between first line, upper level and middle level married and single employees in relation to conflict management styles (Table 7). 
Table 7

Comparison of Different Positions with Marital Status in Relation to Conflict Management Styles

\begin{tabular}{|c|c|c|c|c|c|c|}
\hline $\begin{array}{c}\text { CONFLICT } \\
\text { MANAGEMENT } \\
\text { STYLES }\end{array}$ & $\begin{array}{l}\text { MARITAL } \\
\text { STATUS }\end{array}$ & Mean & $\begin{array}{c}\text { Stand. } \\
\text { Dev. }\end{array}$ & $\mathbf{T}$ & df & Sig. \\
\hline \multicolumn{7}{|c|}{ UPPER LEVEL } \\
\hline \multirow{2}{*}{ COLLABORATION } & MARRIED & 2.6487 & .84376 & \multirow{2}{*}{.772} & \multirow{2}{*}{132.279} & \multirow{2}{*}{.442} \\
\hline & SINGLE & 2.5528 & .88588 & & & \\
\hline \multirow{2}{*}{ ACCOMMODATION } & MARRIED & 3.6551 & 1.01208 & \multirow{2}{*}{-.740} & \multirow{2}{*}{144.551} & \multirow{2}{*}{.461} \\
\hline & SINGLE & 3.7583 & .96352 & & & \\
\hline \multirow{2}{*}{ COMPETITION } & MARRIED & 3.0433 & 1.04154 & \multirow{2}{*}{.490} & \multirow{2}{*}{126.470} & \multirow{2}{*}{.625} \\
\hline & SINGLE & 2.9653 & 1.15163 & & & \\
\hline \multirow{2}{*}{ AVOIDANCE } & MARRIED & 3.5048 & 1.04976 & \multirow{2}{*}{-.912} & \multirow{2}{*}{141.425} & \multirow{2}{*}{.363} \\
\hline & SINGLE & 3.6389 & 1.02340 & & & \\
\hline \multicolumn{7}{|c|}{ MIDDLE LEVEL } \\
\hline \multirow{2}{*}{ COLLABORATION } & MARRIED & 2.6303 & .89986 & \multirow{2}{*}{1.270} & \multirow{2}{*}{609.408} & \multirow{2}{*}{.204} \\
\hline & SINGLE & 2.5405 & .86049 & & & \\
\hline \multirow{2}{*}{ ACCOMMODATION } & MARRIED & 3.7051 & 1.03793 & \multirow{2}{*}{.423} & \multirow{2}{*}{614.730} & \multirow{2}{*}{.672} \\
\hline & SINGLE & 3.6699 & 1.03002 & & & \\
\hline \multirow{2}{*}{ COMPETITION } & MARRIED & 2.9663 & 1.15871 & \multirow{2}{*}{.677} & \multirow{2}{*}{612.559} & \multirow{2}{*}{.499} \\
\hline & SINGLE & 2.9041 & 1.13112 & & & \\
\hline \multirow{2}{*}{ AVOIDANCE } & MARRIED & 3.7584 & 1.06262 & \multirow{2}{*}{.263} & 612755 & 702 \\
\hline & SINGLE & 3.7362 & 1.03877 & & 012.155 & .192 \\
\hline & & RST LII & & & & \\
\hline COLU А ROPА TION & MARRIED & 2.6565 & .96854 & 665 & 84008 & 508 \\
\hline COLLABORATION & SINGLE & 2.7701 & .87472 & -.665 & 84.008 & .508 \\
\hline А CCOMMODАTION & MARRIED & 3.5957 & .96008 & 1450 & 87544 & 148 \\
\hline ACCOMMODATION & SINGLE & 3.3448 & .90974 & 1.459 & 87.544 & .148 \\
\hline COMPFTITION & MARRIED & 3.6739 & 1.00259 & 1333 & 03819 & 186 \\
\hline & SINGLE & 3.4282 & 1.02859 & & & .180 \\
\hline AYOIDANCE & MARRIED & 3.7446 & 1.01856 & 1660 & 05667 & 100 \\
\hline AVUIDANCE & SINGLE & 3.4310 & 1.06864 & 1.060 & $95.66 /$ & .100 \\
\hline
\end{tabular}

* The mean difference is significant at the .05 level.

There is no statistically significant difference between employees with children or not at different levels of the organization in relation to conflict management styles (Table 8). 
Table 8

Comparison of Different Positions with Having Children in Relation to Conflict Management Styles

\begin{tabular}{|c|c|c|c|c|c|c|}
\hline $\begin{array}{c}\text { CONFLICT } \\
\text { MANAGEMENT } \\
\text { STYLES }\end{array}$ & $\begin{array}{c}\text { HAVING } \\
\text { CHILDREN }\end{array}$ & Mean & $\begin{array}{l}\text { Stand. } \\
\text { Dev. }\end{array}$ & $\mathbf{t}$ & df & Sig. \\
\hline \multicolumn{7}{|c|}{ UPPER LEVEL } \\
\hline \multirow{2}{*}{ COLLABORATION } & $\begin{array}{c}\text { HAVE } \\
\text { CHILDREN }\end{array}$ & 2.6596 & .88296 & \multirow{2}{*}{1.049} & \multirow{2}{*}{166.808} & \multirow{2}{*}{.296} \\
\hline & $\begin{array}{l}\text { HAVE NO } \\
\text { CHILDREN }\end{array}$ & 2.5377 & .80149 & & & \\
\hline \multirow{2}{*}{ ACCOMMODATION } & $\begin{array}{c}\text { HAVE } \\
\text { CHILDREN }\end{array}$ & 3.6927 & 1.03454 & \multirow{2}{*}{.110} & \multirow{2}{*}{169.459} & \multirow{2}{*}{.913} \\
\hline & $\begin{array}{l}\text { HAVE NO } \\
\text { CHILDREN }\end{array}$ & 3.6779 & .92233 & & & \\
\hline \multirow{2}{*}{ COMPETITION } & $\begin{array}{c}\text { HAVE } \\
\text { CHILDREN }\end{array}$ & 3.0116 & 1.06823 & \multirow{2}{*}{-.137} & \multirow{2}{*}{149.580} & \multirow{2}{*}{.891} \\
\hline & $\begin{array}{l}\text { HAVE NO } \\
\text { CHILDREN }\end{array}$ & 3.0325 & 1.09676 & & & \\
\hline \multirow{2}{*}{ AVOIDANCE } & $\begin{array}{c}\text { HAVE } \\
\text { CHILDREN }\end{array}$ & 3.5497 & 1.06615 & \multirow{2}{*}{.052} & \multirow{2}{*}{162.479} & \multirow{2}{*}{.958} \\
\hline & $\begin{array}{l}\text { HAVE NO } \\
\text { CHILDREN }\end{array}$ & 3.5422 & .99704 & & & \\
\hline \multicolumn{7}{|c|}{ MIDDLE LEVEL } \\
\hline \multirow{2}{*}{ COLLABORATION } & $\begin{array}{c}\text { HAVE } \\
\text { CHILDREN }\end{array}$ & 2.6465 & .92090 & \multirow{2}{*}{1,336} & \multirow{2}{*}{445,298} & \multirow{2}{*}{, 182} \\
\hline & $\begin{array}{l}\text { HAVE NO } \\
\text { CHILDREN }\end{array}$ & 2.5468 & .85444 & & & \\
\hline \multirow{2}{*}{ ACCOMMODATION } & $\begin{array}{c}\text { HAVE } \\
\text { CHILDREN }\end{array}$ & 3.7088 & 1.10143 & \multirow{2}{*}{.394} & \multirow{2}{*}{434.740} & \multirow{2}{*}{.694} \\
\hline & $\begin{array}{l}\text { HAVE NO } \\
\text { CHILDREN }\end{array}$ & 3.6739 & .99277 & & & \\
\hline \multirow{2}{*}{ COMPETITION } & $\begin{array}{c}\text { HAVE } \\
\text { CHILDREN }\end{array}$ & 2.9145 & 1.17885 & \multirow{2}{*}{-.316} & \multirow{2}{*}{455.662} & \multirow{2}{*}{.752} \\
\hline & $\begin{array}{l}\text { HAVE NO } \\
\text { CHILDREN }\end{array}$ & 2.9449 & 1.12453 & & & \\
\hline \multirow{2}{*}{ AVOIDANCE } & $\begin{array}{c}\text { HAVE } \\
\text { CHILDREN }\end{array}$ & 3.7489 & 1.08314 & \multirow{2}{*}{.038} & 154700 & 070 \\
\hline & $\begin{array}{l}\text { HAVE NO } \\
\text { CHILDREN }\end{array}$ & 3.7456 & 1.03084 & & 454.190 & .910 \\
\hline & & RST LII & & & & \\
\hline COLLABORATION & $\begin{array}{c}\text { HAVE } \\
\text { CHILDREN }\end{array}$ & 2.7171 & .90330 & 117 & 77502 & 007 \\
\hline COLLABURA HON & $\begin{array}{l}\text { HAVE NO } \\
\text { CHILDREN }\end{array}$ & 2.7370 & .91240 & -.117 & 11.592 & .901 \\
\hline & $\begin{array}{c}\text { HAVE } \\
\text { CHILDREN }\end{array}$ & 3.5171 & .85671 & & & \\
\hline ACCOMMODATION & $\begin{array}{l}\text { HAVE NO } \\
\text { CHILDREN }\end{array}$ & 3.3935 & .96508 & .738 & 85.952 & .462 \\
\hline & $\begin{array}{c}\text { HAVE } \\
\text { CHILDREN }\end{array}$ & 3.6829 & .92707 & & & \\
\hline COMPETITION & $\begin{array}{l}\text { HAVE NO } \\
\text { CHILDREN }\end{array}$ & 3.4375 & 1.05847 & 1.348 & 87.060 & .181 \\
\hline AVOIDANCF & $\begin{array}{c}\text { HAVE } \\
\text { CHILDREN }\end{array}$ & 3.7073 & 1.01381 & 1251 & 81138 & 215 \\
\hline A. & $\begin{array}{l}\text { HAVE NO } \\
\text { CHILDREN }\end{array}$ & 3.4647 & 1.07454 & 1.201 & 01.100 & .215 \\
\hline
\end{tabular}

* The mean difference is significant at the .05 level. 
There is a statistically significant difference between employees with high school and masters' degrees working at the upper level in relation to collaboration style (Table 9). Employees with masters degrees working at the upper level use this style (mean 2.8360) more than high school graduates (mean 2.3333). There is also a statistically significant difference between employees with high shool, university and master's degree working at the middle level in relation to competition style. Employees with master's (mean 3.1278) and university (mean 2.9837) degree working at the middle level use this style more than high school graduates (mean 2.6549).

Table 9

Comparison of Different Positions with Education in Relation to Conflict Management Styles

\begin{tabular}{|c|c|c|c|c|c|c|c|}
\hline \multirow{2}{*}{$\begin{array}{c}\text { CONFLICT } \\
\text { MANAGEMENT } \\
\text { STYLES }\end{array}$} & \multirow{2}{*}{\multicolumn{2}{|c|}{ EDUCATION }} & \multicolumn{2}{|c|}{ ANOVA } & \multicolumn{3}{|c|}{$\begin{array}{l}\text { MULTIPLE COMPARISONS } \\
\text { (Scheffe) }\end{array}$} \\
\hline & & & $\mathrm{F}$ & Sig. & Mean Diff. & $\begin{array}{l}\text { Stand. } \\
\text { Dev. }\end{array}$ & Sig. \\
\hline \multicolumn{8}{|c|}{ UPPER LEVEL } \\
\hline \multirow{6}{*}{ COLLABORATION } & \multirow{2}{*}{ High School } & University & \multirow{6}{*}{3.869} & \multirow{6}{*}{.022} & -.28681 & .15328 & .176 \\
\hline & & Master & & & $-.50267(*)$ & .18071 & .022 \\
\hline & \multirow{2}{*}{ University } & High School & & & .28681 & .15328 & .176 \\
\hline & & Master & & & -.21586 & .13949 & .304 \\
\hline & \multirow{2}{*}{ Master } & High School & & & $.50267(*)$ & .18071 & .022 \\
\hline & & University & & & .21586 & .13949 & .304 \\
\hline \multirow{6}{*}{ ACCOMMODATION } & \multirow{2}{*}{ High School } & University & \multirow{6}{*}{.457} & \multirow{6}{*}{.634} & -.16827 & .18092 & .649 \\
\hline & & Master & & & -.09590 & .21330 & .904 \\
\hline & \multirow{2}{*}{ University } & High School & & & .16827 & .18092 & .649 \\
\hline & & Master & & & .07237 & .16465 & .908 \\
\hline & \multirow{2}{*}{ Master } & High School & & & .09590 & .21330 & .904 \\
\hline & & University & & & -.07237 & .16465 & .908 \\
\hline \multirow{6}{*}{ COMPETITION } & \multirow{2}{*}{ High School } & University & \multirow{6}{*}{.131} & \multirow{6}{*}{.877} & -.05317 & .19565 & .964 \\
\hline & & Master & & & .03359 & .23067 & .989 \\
\hline & \multirow{2}{*}{ University } & High School & & & .05317 & .19565 & .964 \\
\hline & & Master & & & .08676 & .17805 & .888 \\
\hline & \multirow{2}{*}{ Master } & High School & & & -.03359 & .23067 & .989 \\
\hline & & University & & & -.08676 & .17805 & .888 \\
\hline \multirow{6}{*}{ AVOIDANCE } & \multirow{2}{*}{ High School } & University & \multirow{6}{*}{.447} & \multirow{6}{*}{.640} & .17870 & .18912 & .640 \\
\hline & & Master & & & .13590 & .22297 & .831 \\
\hline & \multirow{4}{*}{ University } & High School & & & -.17870 & .18912 & 640 \\
\hline & & Master & & & -.04281 & .17211 & .970 \\
\hline & & High School & & & -.13590 & .22297 & .831 \\
\hline & & University & & & .04281 & .17211 & .970 \\
\hline & & MIDD & E LEV & & & & \\
\hline & High Schol & University & & & -.10148 & .08787 & .514 \\
\hline & Hign Schoor & Master & & & -.14743 & .11994 & .470 \\
\hline COLLABORATION & University & High School & 021 & 300 & .10148 & .08787 & .514 \\
\hline COLLABORATION & Uimersity & Master & .921 & .399 & -.04595 & .10269 & .905 \\
\hline & Master & High School & & & .14743 & .11994 & .470 \\
\hline & Master & University & & & .04595 & .10269 & .905 \\
\hline
\end{tabular}


Table 9 (continued)

\begin{tabular}{|c|c|c|c|c|c|c|c|}
\hline \multirow{6}{*}{ ACCOMMODATION } & \multirow{2}{*}{ High School } & University & \multirow{6}{*}{2.204} & \multirow{6}{*}{.111} & -.10904 & .10295 & .571 \\
\hline & & Master & & & .13138 & .14053 & .646 \\
\hline & \multirow{2}{*}{ University } & High School & & & .10904 & .10295 & .571 \\
\hline & & Master & & & .24042 & .12033 & .137 \\
\hline & \multirow{2}{*}{ Master } & High School & & & -.13138 & .14053 & .646 \\
\hline & & University & & & -.24042 & .12033 & .137 \\
\hline \multirow{6}{*}{ COMPETITION } & \multirow{2}{*}{ High School } & University & \multirow{6}{*}{5.745} & \multirow{6}{*}{.003} & $-.32886(*)$ & .11335 & .015 \\
\hline & & Master & & & $-.47293(*)$ & .15472 & .010 \\
\hline & \multirow{2}{*}{ University } & High School & & & $.32886(*)$ & .11335 & .015 \\
\hline & & Master & & & -.14407 & .13247 & .554 \\
\hline & \multirow{2}{*}{ Master } & High School & & & $.47293(*)$ & .15472 & .010 \\
\hline & & University & & & .14407 & .13247 & .554 \\
\hline \multirow{6}{*}{ AVOIDANCE } & \multirow{2}{*}{ High School } & University & \multirow{6}{*}{1.413} & \multirow{6}{*}{.244} & -.15814 & .10471 & .320 \\
\hline & & Master & & & -.20655 & .14293 & .353 \\
\hline & \multirow{2}{*}{ University } & High School & & & .15814 & .10471 & .320 \\
\hline & & Master & & & -.04841 & .12238 & .925 \\
\hline & \multirow{2}{*}{ Master } & High School & & & .20655 & .14293 & .353 \\
\hline & & University & & & .04841 & .12238 & .925 \\
\hline
\end{tabular}

FIRST LINE

\begin{tabular}{|c|c|c|c|c|c|c|c|}
\hline \multirow{6}{*}{ COLLABORATION } & \multirow{2}{*}{ High School } & University & \multirow{6}{*}{2.058} & \multirow{6}{*}{.132} & .31084 & .16133 & .160 \\
\hline & & Master & & & .38933 & .41525 & .645 \\
\hline & \multirow{2}{*}{ University } & High School & & & -.31084 & .16133 & .160 \\
\hline & & Master & & & .07849 & .42060 & .983 \\
\hline & \multirow{2}{*}{ Master } & High School & & & -.38933 & .41525 & .645 \\
\hline & & University & & & -.07849 & .42060 & .983 \\
\hline \multirow{6}{*}{ ACCOMMODATION } & \multirow{2}{*}{ High School } & University & \multirow{6}{*}{1.770} & \multirow{6}{*}{.174} & .11421 & .16620 & .790 \\
\hline & & Master & & & .78667 & .42778 & .188 \\
\hline & \multirow{2}{*}{ University } & High School & & & -.11421 & .16620 & .790 \\
\hline & & Master & & & .67245 & .43329 & .303 \\
\hline & \multirow{2}{*}{ Master } & High School & & & -.78667 & .42778 & .188 \\
\hline & & University & & & -.67245 & .43329 & .303 \\
\hline \multirow{6}{*}{ COMPETITION } & \multirow{2}{*}{ High School } & University & \multirow{6}{*}{.434} & \multirow{6}{*}{649} & -.03050 & .18430 & .986 \\
\hline & & Master & & & .41667 & .47437 & .681 \\
\hline & \multirow{2}{*}{ University } & High School & & & .03050 & .18430 & .986 \\
\hline & & Master & & & .44717 & .48048 & .649 \\
\hline & \multirow{2}{*}{ Master } & High School & & & -.41667 & .47437 & .681 \\
\hline & & University & & & -.44717 & .48048 & .649 \\
\hline \multirow{6}{*}{ AVOIDANCE } & \multirow{2}{*}{ High School } & University & \multirow{6}{*}{.470} & \multirow{6}{*}{.626} & .12220 & . 19069 &. .815 \\
\hline & & Master & & & .40333 & .49081 & .714 \\
\hline & \multirow{2}{*}{ University } & High School & & & -.12220 & . 19069 & .815 \\
\hline & & Master & & & .28113 & .49713 & .852 \\
\hline & \multirow{2}{*}{$\begin{array}{c}\text { Master } \\
\text { High School }\end{array}$} & High School & & & -.40333 & .49081 & .714 \\
\hline & & University & & & -.28113 & .49713 & .852 \\
\hline
\end{tabular}

* The mean difference is significant at the .05 level.

There is a statistically significant difference between employees with 6-15 years total tenure and 1-5 years total tenure working at the first line in relation to avoidance style (Table 10). Employees with 6-15 years total tenure in work life (mean 3.9231) working at the first line use this style more than employees with 1-5 years of total tenure (mean 3.3056). 
Table 10

Comparison of Different Positions with Total Tenure in Worklife in Relation to Conflict Management Styles

\begin{tabular}{|c|c|c|c|c|c|c|c|}
\hline \multirow{2}{*}{$\begin{array}{c}\text { CONFLICT } \\
\text { MANAGEMENT } \\
\text { STYLES }\end{array}$} & \multirow{2}{*}{\multicolumn{2}{|c|}{$\begin{array}{l}\text { TOTAL TENURE IN } \\
\text { WORKLIFE }\end{array}$}} & \multicolumn{2}{|c|}{ ANOVA } & \multicolumn{3}{|c|}{$\begin{array}{c}\text { MULTIPLE COMPARISONS } \\
\text { (Scheffe) }\end{array}$} \\
\hline & & & $\mathrm{F}$ & Sig. & Mean Diff. & $\begin{array}{l}\text { Stand. } \\
\text { Dev. }\end{array}$ & Sig. \\
\hline \multicolumn{8}{|c|}{ UPPER LEVEL } \\
\hline \multirow{6}{*}{ COLLABORATION } & \multirow{2}{*}{ 1-5 Years } & 6-15 Years & \multirow{6}{*}{.698} & \multirow{6}{*}{.499} & -.19721 & .17139 & .517 \\
\hline & & 16-+ Years & & & -.11048 & .16739 & .804 \\
\hline & \multirow{2}{*}{ 6-15 Years } & 1-5 Years & & & .19721 & .17139 & .517 \\
\hline & & 16-+ Years & & & .08673 & .12395 & .783 \\
\hline & \multirow{2}{*}{ 16-+ Years } & 1-5 Years & & & .11048 & .16739 & .804 \\
\hline & & 6-15 Years & & & -.08673 & .12395 & .783 \\
\hline \multirow{6}{*}{ ACCOMMODATION } & \multirow{2}{*}{ 1-5 Years } & 6-15 Years & \multirow{6}{*}{.279} & \multirow{6}{*}{.756} & .13961 & .19968 & .783 \\
\hline & & 16-+ Years & & & .06476 & .19503 & .946 \\
\hline & \multirow{2}{*}{ 6-15 Years } & 1-5 Years & & & -.13961 & .19968 & .783 \\
\hline & & 16-+ Years & & & -.07485 & .14441 & .874 \\
\hline & \multirow{2}{*}{ 16-+ Years } & 1-5 Years & & & -.06476 & .19503 & .946 \\
\hline & & 6-15 Years & & & .07485 & .14441 & .874 \\
\hline \multirow{6}{*}{ COMPETITION } & \multirow{2}{*}{ 1-5 Years } & 6-15 Years & \multirow{6}{*}{.059} & \multirow{6}{*}{.942} & .00568 & .21584 & 1.000 \\
\hline & & 16-+ Years & & & -.04524 & .21081 & .977 \\
\hline & \multirow{2}{*}{ 6-15 Years } & 1-5 Years & & & -.00568 & .21584 & 1.000 \\
\hline & & 16-+ Years & & & -.05092 & .15610 & .948 \\
\hline & \multirow{2}{*}{ 16-+ Years } & 1-5 Years & & & .04524 & .21081 & .977 \\
\hline & & 6-15 Years & & & .05092 & .15610 & .948 \\
\hline \multirow{6}{*}{ AVOIDANCE } & \multirow{2}{*}{ 1-5 Years } & 6-15 Years & & & -.12508 & .20872 & .836 \\
\hline & & 16-+ Years & & & -.02857 & .20386 & .990 \\
\hline & & 1-5 Years & & & .12508 & .20872 & .836 \\
\hline & 6-15 Years & 16-+ Years & .276 & .759 & .09651 & .15095 & .815 \\
\hline & & 1-5 Years & & & .02857 & .20386 & .990 \\
\hline & $16-+$ Years & 6-15 Years & & & -.09651 & .15095 & .815 \\
\hline & & MIDI & LEV] & & & & \\
\hline & 1-5 Years & 6-15 Years & & & .01249 & .08001 & .988 \\
\hline & 1-5 Years & 16-+ Years & & & .08405 & .09367 & .669 \\
\hline COLLARORATION & 6-15Years & 1-5 Years & 420 & 657 & -.01249 & .08001 & .988 \\
\hline СОLLADUKATION & 0-15 Years & 16-+ Years & .420 & (05/ & .07156 & .09826 & .767 \\
\hline & $16-+$ Years & 1-5 Years & & & -.08405 & .09367 & .669 \\
\hline & $10-+$ Years & 6-15 Years & & & -.07156 & .09826 & .767 \\
\hline & 1-5Years & 6-15 Years & & & .01269 & .09364 & .991 \\
\hline & $1-5$ Y Years & 16-+ Years & & & -.21956 & .10962 & .135 \\
\hline АCCOMMODАTON & 6-15Years & 1-5 Years & 2447 & 087 & -.01269 & .09364 & .991 \\
\hline ACC OMIVIODA TION & 0-15 Y rears & 16-+ Years & 2.441 & .081 & -.23225 & .11499 & .131 \\
\hline & $16+$ Years & 1-5 Years & & & .21956 & .10962 & .135 \\
\hline & $10-+$ Years & 6-15 Years & & & .23225 & .11499 & .131 \\
\hline
\end{tabular}


Table 10 (continued)

\begin{tabular}{|c|c|c|c|c|c|c|c|}
\hline \multirow{6}{*}{ COMPETITION } & \multirow{2}{*}{ 1-5 Years } & 6-15 Years & \multirow{6}{*}{1.957} & \multirow{6}{*}{.142} & -.06189 & .10376 & .837 \\
\hline & & 16-+ Years & & & -.23972 & .12146 & .143 \\
\hline & \multirow{2}{*}{ 6-15 Years } & 1-5 Years & & & .06189 & .10376 & .837 \\
\hline & & 16-+ Years & & & -.17783 & .12742 & .378 \\
\hline & \multirow{2}{*}{ 16-+ Years } & 1-5 Years & & & .23972 & .12146 & .143 \\
\hline & & 6-15 Years & & & .17783 & .12742 & .378 \\
\hline \multirow{6}{*}{ AVOIDANCE } & \multirow{2}{*}{ 1.5 Years } & 6-15 Years & \multirow{6}{*}{.978} & \multirow{6}{*}{.377} & .02058 & .09534 & .977 \\
\hline & & 16-+ Years & & & -.13449 & .11161 & .484 \\
\hline & \multirow{2}{*}{ 6-15 Years } & 1-5 Years & & & -.02058 & .09534 & .977 \\
\hline & & 16-+ Years & & & -.15507 & .11708 & .417 \\
\hline & \multirow{2}{*}{ 16-+ Years } & 1-5 Years & & & .13449 & .11161 & .484 \\
\hline & & 6-15 Years & & & .15507 & .11708 & .417 \\
\hline \multicolumn{8}{|c|}{ FIRST LINE } \\
\hline \multirow{6}{*}{ COLLABORATION } & \multirow{2}{*}{ 1-5 Years } & 6-15 Years & \multirow{6}{*}{1.400} & \multirow{6}{*}{.250} & -.00085 & .17963 & 1.000 \\
\hline & & 16-+ Years & & & .35253 & .22009 & .281 \\
\hline & \multirow{2}{*}{ 6-15 Years } & 1-5 Years & & & .00085 & .17963 & 1.000 \\
\hline & & 16-+ Years & & & .35338 & .24090 & .344 \\
\hline & \multirow{2}{*}{ 16-+ Years } & 1-5 Years & & & -.35253 & .22009 & .281 \\
\hline & & 6-15 Years & & & -.35338 & .24090 & .344 \\
\hline \multirow{6}{*}{ ACCOMMODATION } & \multirow{2}{*}{ 1-5 Years } & 6-15 Years & \multirow{6}{*}{2.724} & \multirow{6}{*}{.069} & -.40791 & .18284 & .087 \\
\hline & & 16-+ Years & & & -.29066 & .22402 & .433 \\
\hline & \multirow{2}{*}{ 6-15 Years } & 1.5 Years & & & .40791 & .18284 & .087 \\
\hline & & 16-+ Years & & & .11725 & .24520 & .892 \\
\hline & \multirow{2}{*}{ 16-+ Years } & 1-5 Years & & & .29066 & .22402 & .433 \\
\hline & & 6-15 Years & & & -.11725 & .24520 & .892 \\
\hline \multirow{6}{*}{ COMPETITION } & \multirow{2}{*}{ 1-5 Years } & 6-15 Years & & & -.44017 & .20120 & .095 \\
\hline & & 16-+ Years & & & -.13889 & .24652 & .853 \\
\hline & & 1-5 Years & & & .44017 & .20120 & .095 \\
\hline & 6-15 Years & 16-+ Years & 2.395 & .095 & .30128 & .26983 & .538 \\
\hline & & 1-5 Years & & & .13889 & .24652 & .853 \\
\hline & 16-+ Years & 6-15 Years & & & -.30128 & .26983 & .538 \\
\hline & 1-5 Years & 6-15 Years & & & $-.61752(*)$ & .20486 & .012 \\
\hline & $1-5$ Years & 16-+ Years & & & -.31944 & .25100 & .447 \\
\hline A YOINANE & 615Yors & 1-5 Years & 1634 & & $.61752(*)$ & .20486 & .012 \\
\hline AVOIDANCE & 6-15 Years & 16-+ Years & 4.034 & .011 & .29808 & .27473 & .557 \\
\hline & $16-+$ Years & 1-5 Years & & & .31944 & .25100 & .447 \\
\hline & 10-+ years & 6-15 Years & & & -.29808 & .27473 & .557 \\
\hline
\end{tabular}

* The mean difference is significant at the .05 level.

There is a statistically significant difference between employees with 6-15 years tenure and 1-5 years tenure in the current organization working at the upper level in relation to accommodation style (Table 11). Employees with 1-5 years tenure in the current organization working at the upper level (mean 3.9368) use this style more than employees with 6-15 years of tenure in the current organization (mean 3.3884). 
Table 11

Comparison of Different Positions with Total Tenure in Current Organization in Relation to Conflict Management Styles

\begin{tabular}{|c|c|c|c|c|c|c|c|}
\hline \multirow{2}{*}{$\begin{array}{l}\text { CONFLICT } \\
\text { MANAGEMENT } \\
\text { STYLES }\end{array}$} & \multirow{2}{*}{\multicolumn{2}{|c|}{$\begin{array}{l}\text { TOTAL TENURE } \\
\text { IN CURRENT } \\
\text { ORGANIZATION }\end{array}$}} & \multicolumn{2}{|c|}{ ANOVA } & \multicolumn{3}{|c|}{$\begin{array}{l}\text { MULTIPLE COMPARISONS } \\
\text { (Scheffe) }\end{array}$} \\
\hline & & & $\mathrm{F}$ & Sig. & Mean Diff. & $\begin{array}{l}\text { Stand. } \\
\text { Dev. }\end{array}$ & Sig. \\
\hline \multicolumn{8}{|c|}{ UPPER LEVEL } \\
\hline \multirow{6}{*}{ COLLABORATION } & \multirow{2}{*}{ 1-5 Years } & 6-15 Years & \multirow{6}{*}{.871} & \multirow{6}{*}{.420} & -.16636 & .12756 & .429 \\
\hline & & 16-+ Years & & & -.05048 & .15283 & .947 \\
\hline & \multirow{2}{*}{ 6-15 Years } & 1-5 Years & & & .16636 & .12756 & .429 \\
\hline & & 16-+ Years & & & .11588 & .15545 & .758 \\
\hline & \multirow{2}{*}{ 16-+ Years } & 1-5 Years & & & .05048 & .15283 & .947 \\
\hline & & 6-15 Years & & & -.11588 & .15545 & .758 \\
\hline \multirow{6}{*}{ ACCOMMODATION } & \multirow{2}{*}{ 1-5 Years } & 6-15 Years & \multirow{6}{*}{7.284} & \multirow{6}{*}{.001} & $.54847(*)$ & .14431 & .001 \\
\hline & & 16-+ Years & & & .20493 & .17291 & .497 \\
\hline & \multirow{2}{*}{ 6-15 Years } & 1-5 Years & & & $-.54847(*)$ & .14431 & .001 \\
\hline & & 16-+ Years & & & -.34354 & .17587 & .151 \\
\hline & \multirow{2}{*}{ 16-+ Years } & 1-5 Years & & & -.20493 & .17291 & .497 \\
\hline & & 6-15 Years & & & .34354 & .17587 & .151 \\
\hline \multirow{6}{*}{ COMPETITION } & \multirow{2}{*}{ 1.5 Years } & 6-15 Years & \multirow{6}{*}{.197} & \multirow{6}{*}{.821} & -.08654 & .16066 & .865 \\
\hline & & 16-+ Years & & & -.09804 & .19249 & .878 \\
\hline & \multirow{2}{*}{ 6-15 Years } & 1-5 Years & & & .08654 & .16066 & .865 \\
\hline & & 16-+ Years & & & -.01150 & .19580 & .998 \\
\hline & \multirow{2}{*}{ 16-+ Years } & 1-5 Years & & & .09804 & .19249 & .878 \\
\hline & & 6-15 Years & & & .01150 & .19580 & .998 \\
\hline \multirow{6}{*}{ AVOIDANCE } & \multirow{2}{*}{ 1-5 Years } & 6-15 Years & & & .24416 & .15452 & .289 \\
\hline & & 16-+ Years & & & .26920 & .18513 & .349 \\
\hline & 15 Veanc & 1-5 Years & 1655 & 103 & -.24416 & .15452 & .289 \\
\hline & 6-15 Years & 16-+ Years & 1.655 & .193 & .02505 & .18831 & .991 \\
\hline & & 1-5 Years & & & -.26920 & .18513 & .349 \\
\hline & 16-+ Years & 6-15 Years & & & -.02505 & .18831 & .991 \\
\hline & & MIDD & LEVI & & & & \\
\hline & & 6-15 Years & & & .09674 & .08111 & .491 \\
\hline & 1-5 Years & 16-+ Years & & & -.06751 & .15277 & .907 \\
\hline COLLABORATION & 6-15 Years & 1-5 Years & 006 & 405 & -.09674 & .08111 & .491 \\
\hline COLLABORATION & 6-15 Years & 16-+ Years & .906 & .405 & -.16425 & .16206 & .599 \\
\hline & $16+$ Years & 1-5 Years & & & .06751 & .15277 & .907 \\
\hline & 10-+ Years & 6-15 Years & & & .16425 & .16206 & .599 \\
\hline & & 6-15 Years & & & -.08950 & .09524 & .643 \\
\hline & 1-5 Years & 16-+ Years & & & -.18050 & .17940 & .603 \\
\hline А ССОММОDАTION & 6 15Years & 1-5 Years & 826 & 138 & .08950 & .09524 & .643 \\
\hline ACCOMIOUDATIN & 6-15 Years & 16-+ Years & .820 & .438 & -.09100 & .19031 & .892 \\
\hline & & 1-5 Years & & & .18050 & .17940 & .603 \\
\hline & 16-+ Years & 6-15 Years & & & .09100 & .19031 & .892 \\
\hline
\end{tabular}


Table 11 (continued)

\begin{tabular}{|c|c|c|c|c|c|c|c|}
\hline \multirow{6}{*}{ COMPETITION } & \multirow{2}{*}{ 1-5 Years } & 6-15 Years & \multirow{6}{*}{4.177} & \multirow{6}{*}{.016} & -.23129 & .10488 & .089 \\
\hline & & 16-+ Years & & & -.42931 & .19756 & .095 \\
\hline & \multirow{2}{*}{ 6-15 Years } & 1-5 Years & & & .23129 & .10488 & .089 \\
\hline & & 16-+ Years & & & -.19802 & .20958 & .640 \\
\hline & \multirow{2}{*}{ 16-+ Years } & 1-5 Years & & & .42931 & .19756 & .095 \\
\hline & & 6-15 Years & & & .19802 & .20958 & .640 \\
\hline \multirow{6}{*}{ AVOIDANCE } & \multirow{2}{*}{ 1-5 Years } & 6-15 Years & \multirow{6}{*}{.833} & \multirow{6}{*}{.435} & -.08776 & .09674 & .663 \\
\hline & & 16-+ Years & & & -.18992 & .18223 & .581 \\
\hline & \multirow{2}{*}{ 6-15 Years } & 1-5 Years & & & .08776 & .09674 & .663 \\
\hline & & 16-+ Years & & & -.10216 & .19331 & .870 \\
\hline & \multirow{2}{*}{ 16-+ Years } & 1.5 Years & & & .18992 & .18223 & .581 \\
\hline & & 6-15 Years & & & .10216 & .19331 & .870 \\
\hline \multicolumn{8}{|c|}{ FIRST LINE } \\
\hline \multirow{6}{*}{ COLLABORATION } & \multirow{2}{*}{ 1-5 Years } & 6-15 Years & \multirow{6}{*}{1.723} & \multirow{6}{*}{.183} & .30317 & .19904 & .317 \\
\hline & & 16-+ Years & & & .37856 & .29935 & .452 \\
\hline & \multirow{2}{*}{ 6-15 Years } & 1-5 Years & & & -.30317 & .19904 & .317 \\
\hline & & 16-+ Years & & & .07538 & .33538 & .975 \\
\hline & \multirow{2}{*}{ 16-+ Years } & 1-5 Years & & & -.37856 & .29935 & .452 \\
\hline & & 6-15 Years & & & -.07538 & .33538 & .975 \\
\hline \multirow{6}{*}{ ACCOMMODATION } & \multirow{2}{*}{ 1-5 Years } & 6-15 Years & \multirow{6}{*}{1.050} & \multirow{6}{*}{.353} & -.19659 & .20565 & .634 \\
\hline & & 16-+ Years & & & -.37505 & .30928 & .481 \\
\hline & \multirow{2}{*}{ 6-15 Years } & 1-5 Years & & & .19659 & .20565 & .634 \\
\hline & & 16-+ Years & & & -.17846 & .34650 & .876 \\
\hline & \multirow{2}{*}{ 16-+ Years } & 1-5 Years & & & .37505 & .30928 & .481 \\
\hline & & 6-15 Years & & & .17846 & .34650 & .876 \\
\hline \multirow{6}{*}{ COMPETITION } & \multirow{2}{*}{ 1-5 Years } & 6-15 Years & & & -.23920 & .22609 & .573 \\
\hline & & 16-+ Years & & & .20696 & .34002 & .831 \\
\hline & 615 Yoom & 1-5 Years & 052 & 420 & .23920 & .22609 & .573 \\
\hline & 6-15 Years & 16-+ Years & .852 & .429 & .44615 & .38095 & .505 \\
\hline & & 1.5 Years & & & -.20696 & .34002 & .831 \\
\hline & 16-+ Years & 6-15 Years & & & -.44615 & .38095 & .505 \\
\hline & 1-5 Years & 6-15 Years & & & -.33862 & .23364 & .353 \\
\hline & 1-5 Years & 16-+ Years & & & -.05593 & .35137 & .987 \\
\hline Y YOIDANCF & 615 Years & 1-5 Years & 1051 & 252 & .33862 & .23364 & .353 \\
\hline AVOIDANCE & 6-15 Years & 16-+ Years & 1.051 & .352 & .28269 & .39367 & .773 \\
\hline & & 1-5 Years & & & .05593 & .35137 & .987 \\
\hline & 16-+ Years & 6-15 Years & & & -.28269 & .39367 & .773 \\
\hline
\end{tabular}

* The mean difference is significant at the .05 level. 


\section{CONCLUSION AND DISCUSSION}

The results of the study reveal that demographics are not significantly related to conflict management styles although there exist some differences between different groups.

\section{Summary of the Findings}

One important result of the study is the use of different conflict management styles according to the position of the employees. First level employees were found to use the competition style more than the upper and the middle level managers.

The second finding reveals that conflict management style differs according to the total tenure in work life and tenure in the current organization. Those employees with longer tenures in work life were found to use the accommodating style more and those employees with longer tenures both in work life and in the current organization use the competing style more. The third finding of the study is that conflict management styles do not differ according to marital status, having children and education.

However, the results of the study conducted by Yağcioğlu (1997) point out that those employees between 18-25 of age and had either high school or lower education preferred to use accomodating styles. On the other hand, Akbaş (2001) found a relationship between both tenure and gender and the conflict management styles, but no relationship between conflict management styles and marital status.

The findings of this study are similar to those of previous studies. The literature on conflict management styles of males and females with management and nonmanagement positions has provided inconsistent results. For example, Mc Kenna and Richardson (1995) found Singaporean male managers use the compromising style more than women, while female managers were more inclined to use the avoiding style. The research conducted by Chan et al. (2006) showed that males and females did not differ signifcantly in terms of using conflict management styles. However, the results of t-tests revealed that females tend to avoid conflicts significantly more than males and males prefer the dominant style more than females. Another study carried out by Çetin and Hacıfazlıoğlu (2004) found that female faculty members favored avoidance more significantly than their male counterparts, but no significant difference was found between female and male teachers.

In research conducted by Canlı (2001), a difference between male and female subordinates was found in terms of conflict management styles. This study indicated that females used avoidance style more than male subordinates. Furthermore, in another study, male managers were found to use mostly the compromising style and female managers were found to use the dominating style (Ünver, 2002). While Rahim (1983) found that women were more likely to use cooperative styles (such as obliging and integrating), Bedel and Sistrunk (1973) claimed that women were more competitive. The moderate to high level of cooperativeness via the integrating scale seemed to be responsible for reducing the difference between the conflict management styles of the two genders.

Socially appropriate behavior is different for females and males in many countries around the world; therefore, it is possible to assume that females and males would prefer to resolve conflicts with different conflict style choices (Shockley and Zalabak, 1981). In the United States, historically, males have been socialized to communicate in direct, confrontational ways, assuming the dominant power 
position; females have been socialized to take care of others, and play a more receptive role (Gilligan, 1977; Stockard and Lach, 1990; Zammuto et al., 1979). Kolb (1993: 139) states:

Existing research and our own experience suggest that the voices of women are often hushed in formal negotiation. Conflict and competition are important in formal negotiation, and therefore, it may not be a comfortable place for many women.

With such difference, styles such as competing or collaboration have been assumed to be popular choices for males on conflict resolution self-report instruments (Mills and Chusmir, 1988). Females, for whom relationships may be more important, and for whom aggressive behavior is less forgiven (Ting-Toomey, 1986), would seem more likely to prefer such styles as accomodating and withdrawing, and compromising. As Ting-Toomey (1986: 79) states, "Males typically engage in more direct, "upfront' strategies. Females typically engage in either indirect, 'smoothing' communication strategies to diffuse the conflict topic, or engage in avoidance or withdrawal strategies."

Furthermore, Brewer et al. (2002) suggest that although biological sex may be related to conflict management styles, gender role is more important in determining the styles of conflict management. The researchers state that masculine and feminine characteristics are learned, especially when individuals progress in an organization. Previous studies asking whether different genders have different conflict management styles have come up with contradictory results. Rubin and Brown (1975) found that women have a cooperative approach to conflict whereas men have a more competitive one. Studies conducted by Ilmer (1980), Kilman and Thomas (1975), Ruble and Stander ( 1990) and Rosenthal and Hautaluoma (1988) found that women prefer accommodation and compromise more and domination and competition less than men.

However, the results of this study revealed that middle level female managers utilize the accommodation style more than upper level female managers and first line female managers use the competition style more than middle level female managers. The middle level female managers were found to use the avoidance style more than first line female managers. On the other hand, first line male managers were found to use a competition style more than middle level and upper level male managers.

The results of the study conducted by Brewer et al. (2002) revealed a significant difference between upper and lower status employees in relation to conflict management styles. There was a significant difference between upper and lower status employees in relation to accommodation style. Lower status employees were found to be more accomodating than their upper status counterparts. Upper organizational status employees were found to prefer an integrating (collaborating) style, but not the dominating (competing) style. The explanation brought for this finding is that people at the upper organizational levels experience particular types of conflict that have taught them to realize and put into use various types of solutions. Portello and Long (1994) suggest that since executive training programs emphasize collaborative attempts at problem-solving, and upper-level organizational roles requiring creative and collaborative problem-solving approaches, might be the reason for these results. These results contradict the findings of the current study since in this study, the lower status employees were found to utilize the competition style more than the middle and upper status employees.

Theoretically, given power differences, superiors are generally expected to prefer problem-solving, compromising and forcing; peers are expected to be less aggressive with superiors than each other, 
but more so with subordinates; and subordinates are predicted to tend toward the least aggressive styles, withdrawing and smoothing (Mills and Chusmir, 1988; Musser, 1982; Oetzel, 1998; Rahim and Buntzman, 1989; Renwick, 1975; and Renwick; 1977). That is, given the inherent power differences, a subordinate may not be willing to utilize any conflict style to oppose a superior, while a superior may have more freedom to use aggressive styles, particularly in order to meet company production goals (Rahim and Buntzman, 1989). Peers are considered most likely to use compromise with each other, given the equality of power.

Research would appear to bear organizational role predictions out to a certain extent (Mills and Chusmir, 1988; Musser, 1982; Oetzel, 1998; Phillips and Cheston, 1979; Rahim and Buntzman, 1989). For example, Phillips and Cheston (1979) studied business managers and found that superiors were more likely to choose the use of competition with subordinates than vice versa, while compromise was the style most likely to be prefered for use with one's peers. Several researchers have found subordinates to prefer the styles of withdrawing or accomodating when in conflict with superiors, perhaps because of the risk of negative consequences, such as job loss (Kahn et al., 1964; Phillips and Cheston, 1979; Rahim, 1986). However, Renwick's (1975) research with U.S. business companies found the top three styles for superiors to be problem-solving, compromising and smoothing, contrary to theorized predictions. Renwick also measured subordinates' conflict style rankings, finding the top three styles to be compromising, problem-solving, and competing. Similarly, Rahim (1983) indicated that subordinates most likely prefer the use of collaboration and competition. Likewise, Canl1 (2001) found that employees with non-managerial positions used a dominating style more than employees with managerial positions. Paulson's (1986) research with middle managers from the United States, on the other hand, found no significant differences. Research conducted by Slabbert (2004) showed that there would be significant differences in the modes of conflict resolution at different organizational levels within traditional organizations. It is apparent that the styles of conflict resolution relate to the existence of a strict organizational hierarchy, with very little deviation within both organizational groupings, i.e., middle and junior managers. In summary, middle managers utilize high assertiveness/low cooperation styles, while junior managers demonstrate directly opposite styles, i.e., low assertiveness/high cooperation. Ünver (2002) indicated that both subordinates and superiors used mostly a collaborating style when they managed conflict. Among managers there is a difference between age groups in terms of collaborating, competing and compromising styles, but no difference in terms of avoiding style.

In a study by Cavanagh (1991), comparisons were made between the conflict management, styles of staff nurses and nurse managers and scores obtained by staff nurses on avoidance were compared with similar scores from nurse managers. The results showed that in no comparisons were any statistically significant differences obtained. In Yağcioğlu's study (1997) subordinates were found to use avoiding styles more than upper level and middle level managers. On the other hand, managers prefered competition styles more than the others.

The current study found that there is a correlation between tenure in work life and accommodation and tenure in current organization and competition. The previous research findings by Sorenson and Hawkin (1995), however, found no relationship between the tenure and the preferred conflict management style. 


\section{Managerial Implications}

Although the results of the study revealed some differences in conflict management styles in relation to tenure and position, it is important that for conflicts to be managed functionally, one style may be more appropriate than another depending upon the situation (Rahim, 2001; Rahim and Bonoma, 1979; Thomas, 1977). However, some behavioral scientists suggest that a collaborating or problem-solving style is most appropriate for managing conflict (Blake and Mouton, 1964; Likert and Likert, 1976). In general, collaborating and to some extent compromising styles are appropriate for dealing with strategic issues. The rest of the styles can be used to deal with tactical or day-to-day problems. While collaborating is useful for effectively dealing with complex problems and when one party alone cannot solve the problem and when there is a need in utilizing the skills, information, and other resources possessed by different parties to define or redefine a problem and to formulate effective alternatives, the accomodating style might be useful when a party is not familiar with the issues involved in a conflict or the other party is right and the issue is much more important to the other party and when one believes that preserving the relationship is important. It is not appropriate, however, if the issue involved in a conflict is important to the party and the party believes that he or she is right and when a party believes that the other party is wrong or unethical.

The competing style, on the other hand, might be beneficial when the issues involved in a conflict are important to the party or an unfavorable decision by the other party may be harmful to this party but is inappropriate when the issues involved in the conflict are complex and there is enough time to make a good decision and subordinates have high levels of competence. Avoiding style may be used when the potential dysfunctional effect of confronting the other party outweighs the benefits of the resolution of conflict. It may be inappropriate when the issues are important to a party and when it is the responsibility of the party to make decisions. A compromising style is useful when the goals of the conflicting parties are mutually exclusive or when both parties are equally powerful and when consensus cannot be reached and the parties need a temporary solution to a complex problem, but is inappropriate for dealing with complex problems needing problem-solving approach and if a party is more powerful than another and believes that his or her position is right.

\section{Limitations and Implications for Future Research}

The study included a limited number of people and a limited number of organizations in Istanbul. The results cannot be generalized due to this limitation.

Another limitation is the demographics that have not been included in the study. These excluded demographics (age, place of birth, sector of the organization, number of children, related variables with education such as faculty, high school graduated from, public or private university studied, city of accomodation, number of income earners, income level) could be included in further studies with different sampling methods.

Although the respondents were asked how they behaved when in a conflict, in fact the answer is the individual's behavioral intentions, rather than his or her actual behavior. Sometimes, intentions translate into actual behavior, but sometimes they do not. By asking respondents what they want to do, what they believe they should do, and what they actually do in a conflict might give the opportunity to depict the differences between the intentions and actual behavior. 


\section{REFERENCES}

Akbaş, S. (2001). “Örgütsel Çatışmada Bireysel farklılıkların Önemi ve Hava Trafik Kontrolörleri ile Pilotlar Üzerinde Buna İlişkin Bir Uygulama Çalışması.” Yüksek Lisans Tezi, Anadolu Üniversitesi, Sosyal Bilimler Enstitüsü, Sivil Havacılık Anabilimdalı, Eskişehir.

Akdağ, A.T. (2003). “Örgütlerde Kişilerarası Çatışma, Baş Etme Yöntemleri ve Kişilerarası İlişki Tarzları.” Yüksek Lisans Tezi, İstanbul Üniversitesi, Sosyal Bilimler Enstitüsü, Davranış Bilimleri Anabilimdalı, İstanbul.

Amason, A.C. (1996). "Distinguishing the Effects of Functional and Dysfunctional Conflict on Strategic Decision Making: Resolving a Paradox for Top Management Teams," Academy of Management Journal, 39: 123-148.

Amason, A.C., Thompson, K.R., Hochwarter, W.A., and Harrisson, A.W. (1995). "Conflict: An Important Dimension in Successful Management Teams," Organizational Dynamics, 23: 20-35.

Baxter, L.A. and Shepherd, T.L. (1978). "Sex-role Identity, Sex of Other and Affective Relationship as Determinants on Interpersonal Conflict Management Styles,” Sex Roles, 4: 813-825.

Bedell, J. and Sistrunk, F. (1973). "Power, Opportunity Costs and Sex in a Mixed-motive Game," Journal of Personality and Social Psychology, 31: 634-643.

Bern, S.L. and Lenney, E. (1976). "Sex-typing and the Avoidance of Psychological Androgny, Journal of Personality and Social Psychology," 31: 634-643.

Blake, R.R. and Mouton, J.S. (1964). The Managerial Grid. Houston, TX: Gulf Publishing.

Brenner, O.C., Tomkiewicz, J., and Schein, V.E. (1989). "The Relationship between Sex Role Stereotypes and Requisite Management Characteristics," Academy of Management Journal, 32: 662669.

Brewer, N., Gren, B., Mitchell, P., and Weber, N. (2002). "Gender Role, Organizational Status and Conflict Management Styles," International Journal of Conflict Management, 13(1): 78-94.

Canlı, E. (2001). “Örgütlerde Kişiler Arası Çatışma.” Yüksek Lisans Tezi, İstanbul Üniversitesi, Sosyal Bilimler Enstitüsü, Psikoloji Bölümü, İstanbul.

Cavanagh, S.J.C. (1991). "The Conflict Management Style of Staff Nurses and Nurse Managers," Journal of Advanced Nursing, 16: 1254-1260.

Çetin, M.O. and Hacıfazlıoğlu, O. (2004). "Conflict Management Styles : A Comparative Study of University Academics and High School Teachers," Journal of American Academy of Business, 5(2): 325-332. 
Chan, C.C.A., Monroe, G., Ng, J., and Tan, R. (2006). "Conflict Management Styles of Male and Female Junior Accountants," International Journal of Management, 23(2): 289-295.

Chen, G. and Tjosvold, D. (2002). "Conflict Management and Team Effectiveness in China: The Mediating Role of Justice,” Asia Pacific Journal of Management, 19(4): 557-572.

Cook, E.P. (1985). Psychological Androgyny. New York, NY: Pergamon Press. Cited in Brewer, N., Mitchell, P., and Weber, N. (2002). "Gender Role, Organizational Status and Conflict Management Styles," International Journal of Conflict Management, 13(1):78-94.

Cornille, T.A., Pestle, R.E., and Vanwy, R.W. (1999). "Teachers' Conflict Management Styles," International Journal of Conflict Managemet, 10(1): 287-305.

Coser, L.A. (1956). The Functions of Social Conflict. Glencoe, Illinois: The Free Press. Cited in Cavanagh, S.J.C. (1991). "The Conflict Management Style of Staff Nurses and Nurse Managers," Journal of Advanced Nursing, 16: 1254-1260.

Cosier, R.A., and Ruble, T.L. (1981). "Research on Conflict-handling Behavior. An Experimental Approach,” Academy of Management Journal, 24: 816-831.

Cosier, R.A., and Schwenk, C.R. (1990). "Agreement and Thinking Alike: Ingredients for Poor Decisions," Academy of Management Executive, 4(1): 69-74.

Chusmir, L.H., and Mills, J. (1988). “Resolution of Conflict: Managerial Gender Differences.” Paper presented at the annual meeting of the Academy of Management, Anaheim, CA.

Dahrendorf, R. (1959). Class and Class Conflict in Industrial Society. Standford: Standford University Press. Cited in Cavanagh, S.J.C. (1991). "The Conflict Management Style of Staff Nurses and Nurse Managers," Journal of Advanced Nursing, 16: 1254-1260.

De Dreu, C. and Van de Vliert, E. (eds.) (1997). Using Conflict in Organizations. London: Sage Publications. Cited in Chan, C.C.A., Monroe, G., Ng, J., and Tan, R. (2006). "Conflict Management Styles of Male and Female Junior Accountants,” International Journal of Management, 23(2): 289.

De Dru, C.K.W. and Weingart, L.R. (2003). “Task Versus Relationship Conflict, Team Performance, and Team Member Satisfaction: A Meta Analysis," Journal of Applied Psychology, 88: 741-749.

Deutsch, M. (1971). "Towards an Understanding of Conflict," International Journal of Group Tensions. 1: 42-54.

Dreu, C.K.W., Evers, A., Beersma, B., Kluwer, E.S., and Nauta, A. (2001). “A Theory-Based Measure of Conflict-Management Strategies in the Workplace," Journal of Organizational Behavior, 22 (6): 645- 658.

Drory, A. and Ritu, I. (1997). "Effects of Work Experience and Opponent's Power on Conflict Management Styles," International Journal of Conflict Management, 8(2): 148-161. 
Eagly, A.H. and Johnson, B.T. (1990). "Gender and Leadership Style: A Meta Analysis," Psychological Bulletin," 108: 233-256.

Fagenson, E.A. (1990). "Perceived Masculine and Feminine Attributes Examined as a Function of Individual's Sex and Level in the Organizational Power Hierarchy: A Test of Four Theoretical Perspectives," Journal of Applied Psychology, 75: 204-211.

Gilligan, C. (1977). "In a Different Voice; Women's Conception of the Self and Morality," Harvard Education Review, 47: 481-517.

Gree, B., Brewer, N., Mitchell, P., and Weber, N. (2002). "Gender Role, Organizational Status and Conflict Management Styles," International Journal of Conflict Management, 13(1): 78-94.

Hendel T., Fish, M., and Galon, V. (2005). "Leadership Style and Choice of Strategy in Conflict Management among Israeli Nurse Managers in General Hospitals," Journal of Nursing Management 13: $137-146$.

Ilmer, J.R. (1980). "The Effects of Occupational Category, Organizational Level, Organizational Size, Sex and Conflict Opponent on the Conflict-handling Styles of Hospital Management Personnel." MBA thesis, Youngstown State University.

Jameson, J.K. (1999). "Toward Comprehensive Model for the Assesment and Management of Intraorganizational Conflict: Developing the Framework," International Journal of Conflict Management, 10: 268-294.

Jehn, K.A. (1994). "Enhancing Effectiveness: An Investigation of Advantages and Disadvantages of Value-based Intragroup Conflict," International Journal of Conflict Management, 5: 223-238.

----- (1997). “A Qualitative Analysis of Conflict Types and Dimensions in Organizational Groups," Administrative Science Quarterly, 42: 530-557.

Kahn, R., Wolf, D., Quinn, R., Snoek, J., and Rosenthal, R. (1964). Organizational Stress: Studies in Role Conflict and Ambiguity. New York: Wiley.

Kanter, R.M. (1977). Men and Women of the Corporation. New York: Basic. Cited in Brewer, N., Mitchell, P. and Weber, N. (2002). "Gender Role, Organizational Status and Conflict Management Styles," International Journal of Conflict Management, 13(1): 78-94.

Kelly, J. (1970). “Make Conflict Work for You,” Harvard Business Review, 48: 103-113.

Kilmann, R. H. and Thomas, K.W. (1975). "Interpersonal Conflict-handling Behavior as a Reflection of Jungian Personality Dimensions," Psychological Reports, 37: 971-980.

Kolb, D. A. (1993). LSI-IIa: Self-scoring Inventory and Interpretation Booklet. Boston: McBer and Company. 
Korabik, K., Baril, G.L., and Watson, C. (1993). "Manager's Conflict Management Style and Leadership Effectiveness: The Moderating Effects of Gender," Sex Roles, 29: 405-420.

Kozan, M.K. (1997). "Culture and Conflict Management: A Theoretical Framework, International Journal of Conflict Management," 8 (4): 338-360.

Kuşçuluoğlu, S. (2004). “A Study on Conflict Styles of Individual's in Relation to the Dominant Personality Factors.” MA thesis, Marmara University, Institute of Social Sciences, Faculty of Business Administration. İstanbul.

Lewis, J.H. (1976). “Conflict Management,” Journal of Nursing Administration. 6: 18-22.

Likert, R. and Likert, J.G. (1976). New Ways of Managing Conflict. New York: McGraw-Hill.

Litterer, J.A. (1966). “Conflict in Organizations A Reexamination,” Academy of Management Journal, 9: 178-186.

Mayo, E. (1945). The Social Problems of an Industrial Civilization, Cambridge. Massachusetts: Harvard Universitiy Press. Cited in Cavanagh, S.J.C. (1991). "The Conflict Management Style of Staff Nurses and Nurse Managers," Journal of Advanced Nursing, 16: 1254-1260.

McKenna, S. and Richardson, J. (1995). "Business Values, Management and Conflict Handling: Issues in Contemporary Singapore,” Journal of Management Development, 11(4): 56-70.

Miles, R.H. (1980). Macro Organizational Behavior. Glenview, IL: Scott, Foresman.

Mosca, G. (1939). The Ruling Class. NewYork: McGraw Hill. Cited in Cavanagh, S.J.C. (1991). "The Conflict Management Style of Staff Nurses and Nurse Managers," Journal of Advanced Nursing, 16: 1254-1260.

Musser, S.J. (1982). "A Model for Predicting the Choice of Conflict Management Strategies by Subordinates in High-stakes Conflicts," Organizational Behavior and Human Performance, 29: 257269.

O’Connor, A.B. (1978). "Sources of Conflict for Faculty Members," Journal of Nursing Education, 17(5): 35-38.

Oetzel, J.G., (1998). "The Effects of Self-construals and Ethnicity on Self-reported Conflict Styles," Communication Reports, 11 (2):133-144.

Öztaş, U. (2005). “Örgütsel Çatışma Yönetiminde Cinsiyet Faktörünün Etkisi.” Yüksek Lisans Tezi, Akdeniz Üniversitesi, Sosyal Bilimler Enstitüsü, İşletme Anabilim Dalı, Antalya.

Pearson, A.W., Ensley, M.D., and Amason, A.C. (2002). “An Assessmet and Refinement of Jehn's Intragroup Conflict Scale,” International Journal of Conflict Management, 13: 110-126. 
Phillips, E. and Cheston, R. (1979). “Conflict Resolution: What Works?” California Management Review, 21(4): 76-83.

Pondy, L.R. (1992). "Reflections on Organizational Conflict," Journal of Organizational Behavior, 13: $257-261$.

Portello, J.Y. and Long, B.C. (1994). "Gender Role Orientation, Ethical and Interpersonal Conflicts and Conflict Handling Styles of Female Managers," Sex Roles, 31: 683-701.

Powell, G.N. (1988). Women and Men in Management. Newbury Park, CA: Sage. Cited in Brewer, N., Mitchell, P., and Weber, N. (2002). "Gender Role, Organizational Status and Conflict Management Styles," International Journal of Conflict Management, 13(1).78-94.

Powell, G.N. and Butterfield, D.A. (1979). “The 'Good Manager.' Masculine or Androgynous?” Academy of Management Journal, 22: 395-403.

Priem, R.L., Harrison, D.A., and Muir, N.K. (1995). "Structured Conflict and Consensus Outcomes in Group Decision Making,” Journal of Management, 21(4): 691-710.

Pulhamus, A.R. (1991). "Conflict Handling-A Common Sense Approach to Appraising Supervisory Performance," Public Personnel Management, 20: 485-492.

Rahim, M.A. (1983). “A Measure of Styles Handling Interpersonal Conflict,” Academy of Management Journal, 26(2): 368-376.

------ (1986). Managing Conflict in Organizations. New York: Praeger Publishers.

----- (1992). Managing Conflict in Organizations (2nd ed.). Westport, CT: Praeger.

----- (2000). "Emprical Studies on Handling Conflict," International Journal of Conflict Management, 11: 5-8.

----- (2001). Managing Conflict in Organizations (3rd ed.). Westport, CT: Quorum Books.

----- (2002). “Toward a Theory of Managing Organizational Conflict," International Journal of Conflict Management, 11: 5-8.

Rahim, M.A. and Blum, A.A. (Eds.) (1994). Global Perspectives on Organizational Conflict. Westport, CT: Praeger. Citied in Elsayed, E., Sayed M. and Buda, R. (1996). "Organizational Conflict: A Comparative Analysis of Conflict Styles Across Cultures," International Journal of Conflict Management, 7(1): 71-80.

Rahim, M.A. and Bonoma, T.V. (1979). "Managing Organizational Conflict: A Model for Diagnosis and Intervention," Psychological Reports, 44: 1323-1344. 
Rahim, M.A. and Buntzman, G.F. (1989). "Supervisory Power Bases, Styles of Handling Conflict with Subordinates, and Subordinate Compliance and Satisfaction," Journal of Psychology, 123: 195210.

Rahim, M.A. and Magner, N.R. (1995). "Confirmatory Factor Analysis of the Styles of Handling Interpersonal Conflict: First Order Factor Model and Its Invariance across Groups," Journal of Applied Psychology, 80(1): 122-132.

Raven, B.F. and Kruglanski, A.W. (1970). "Conflict and Power," in P.G. Swingle (ed.), The Structure of Conflict: 69-109. New York, NY: Academic Press.

Renwick, P.A. (1975). "Perception and Management of Superior-subordinate Conflict," Organizational Behavior and Human Performance, 13: 444-456.

(1977). "The Effects of Sex Differences on the Perception and Management of Superiorsubordinate Conflict: An Exploratory Study," Organizational and Human Performance, 19: 403415 .

Roloff, M.E. (1987). "Communication and Conflict," in C. R. Berger and S. H. Chaffee (eds.), Handbook of Communication Science: 484-534. Newbury Park, CA: Sage. Cited in Rahim, M.A. (2002). "Toward a Theory of Managing Organizational Conflict," International Journal of Conflict Management, 13 (3): 206-235.

Rosenthal, D.B. and Hautaluoma, J. (1988). "Effects of Importance of Issues, Gender, and Power of Contenders on Conflict Management Style," Journal of Social Psychology, 128: 699-701.

Rubin, J.Z. and Brown, B.R. (1975). The Social Psychology of Bargaining Negotiation. New York: Academic Press. Cited in Brewer, N., Mitchell, P. and Weber, N. (2002). "Gender Role, Organizational Status and Conflict Management Styles," International Journal of Conflict Management, 13(1): 78-94.

Ruble, T.L. and Stander, N.E. (1990). "Effects of Role and Gender on Conflict-handling Styles." Paper presented at the third conference of the International Association for Conflict Management, Vancouver, BC.

Schmidt, W.H. and Tannenbaum, R. (1960). "The Management of Differences," Harvard Business Review. 38: 107-115.

Shockley-Zalabak, P. (1988). "Assessing the Hall Conflict Management Survey," Management Communication Quarterly, 1: 302-320.

Slabbert, A.D. (2004). "Conflict Management Styles in Traditional Organizations," Social Science Journal, 41: 33-92.

Sorenson, P. and Hawkins, K. (1995). "Gender, Psychological Type and Conflict Style Preference," Management Communication Quarterly, 9: 115-127. 
Stockard, J. and Lach, D. (1990). "Conflict Resolution: Sex and Gender Roles," in J.B. Gitler (ed.), The Annual Review of Conflict Knowledge and Conflict Resolution, 1: 69-99. NY: Garland Publishing Inc.

Sweeney, B and Carruthers, W.L. (1996). "Conflict Resolution: History, Philosophy, Theory, and Educational Applications,” School Counselor, 43(5): 326-344.

Taylor, D.M. and Moghaddam, F.M. (1994). Theories of Intergroup Relations. London: Praeger.

Temkin, T. and Cummings, H.W. (1986). "The Use of Conflict Management Behaviors in Voluntary Organizations: An Exploratory Study,” Nonprofit and Voluntary Sector Quarterly, 15(1): 5-18.

Thomas, K. (1976). "Conflict and Conflict Management," in M. Dunnette (ed.) Handbook of Industrial and Organizational Psychology: 889-935. Chicago: Rand Mcnally, Inc. Cited in Jung, S. (2003). "The Effects of Organizational Culture on Conflict Resolution in Marketing," Journal of American Academy of Business, 3(1/2): 242.

Thomas, K.W. (1977). "Toward Multi-dimensional Values in Teaching: The Example of Conflict Behaviors," Academy of Management Review, 2: 484-490.

Thomas, K.W. and Schmidt, W.H. (1976). “A Survey of Managerial Interests with Respect to Conflict,” Academy of Management Journal, 33(19): 315-318.

Ting-Toomey, S. (1986). "Conflict Styles in Black and White Subjective Cultures," in Y. Kim (ed.), Current Research in Interethnic Communication: Beverly Hills: CA: Sage.

Tjosvold, D. and Sun, H.F. (2002). "Understanding Conflict Avoidance: Relationships, Motivations, Actions, and Consequences," International Journal of Conflict Management, 13(2): 142-164.

Wall, J.A. and Callister, R.R. (1995). "Conflict and Its Management," Journal of Management, 21: $515-558$

Yağcıŏ̆lu, B.D. (1997). “Örgütlerde Çatışma Düzeyleri ve Çatışmanın Ele Alınış Biçimleri,” Yüksek Lisans Tezi, Ege Üniversitesi Sosyal Bilimler Enstitüs Sosyal Psikoloji Anabilim dalı, İzmir.

Zammuto, R.F., London, M., and Rowland, K.M. (1979). "Effects of Sex on Commitment and Conflict Resolution," Journal of Applied Psychology, 64: 227-231 